\title{
On the Hamel Coefficients and the Boltzmann-Hamel Equations for the Rigid Body
}

\author{
Andreas Müller $^{1}$ (1) \\ Received: 7 May 2020 / Accepted: 19 February 2021 / Published online: 19 March 2021 \\ (c) The Author(s) 2021
}

\begin{abstract}
The Boltzmann-Hamel (BH) equations are central in the dynamics and control of nonholonomic systems described in terms of quasi-velocities. The rigid body is a classical example of such systems, and it is well-known that the $\mathrm{BH}$-equations are the Newton-Euler (NE) equations when described in terms of rigid body twists as quasi-velocities. It is further known that the NE-equations are the Euler-Poincaré, respectively, the reduced Euler-Lagrange equations on $S E$ (3) when using body-fixed or spatial representation of rigid body twists. The connection between these equations are the Hamel coefficients, which are immediately identified as the structure constants of $S E$ (3). However, an explicit coordinate-free derivation has not been presented in the literature. In this paper the Hamel coefficients for the rigid body are derived in a coordinate-free way without resorting to local coordinates describing the rigid body motion. The three most relevant choices of quasi-velocities (body-fixed, spatial, and hybrid representation of rigid body twists) are considered. The corresponding BHequations are derived explicitly for the rotating and free floating body. Further, the Hamel equations for nonholonomically constrained rigid bodies are discussed, and demonstrated for the inhomogenous ball rolling on a plane.
\end{abstract}

Keywords Rigid body · Nonholonomic dynamics · Quasi-coordinates ·

Quasi-velocities · Hamel coefficients · Boltzmann-Hamel equations · Lagrange reduction · Euler-Poincaré equations

Mathematics Subject Classification 37J60, 37J39, 70G45, 70G65, 70H33, 37N05, $70 \mathrm{H} 03,53 \mathrm{Z} 30$

Communicated by Jorge Cortes.

Andreas Müller
a.mueller@jku.at
$1 \quad$ JKU Johannes Kepler University, Linz, Austria 


\section{Introduction}

The Boltzmann-Hamel $(\mathrm{BH})$ equations are a milestone result of the research on the dynamics of nonholonomic systems, which was initiated by Hertz (1910). Hamel continued previous works of Volterra (1898) (who continued the work of Beltrami), Tzénoff (1920, 1924) (who built upon the work of Chaplygin (1897a; 1897b; 1911)), Poincaré (1892), Voronets (1901), Woronetz (1910) and Boltzmann (1902) in particular, into a general concept. Key elements in these equations are the three-index symbols that are referred to as 'Hamel coefficients', which reveal the nonholonomic nature of the quasi-coordinates, respectively of the constraints a system is subjected to.

If the Lagrangian is invariant under the action of a Lie group, then the Hamel coefficients are identical to the structure constants of this group. The BH-equations are then the Euler-Lagrange equations corresponding to a reduced variational principle on this symmetry group, respectively, the Euler-Poincaré equations (Bloch et al. 2009; Marsden and Ratiu 2003; Marsden and Scheurle 1993). For this reason the BH-equations are often used as synonym for the reduced Euler-Lagrange equations (Ball and Zenkov 2015; Bloch et al. 2009). The configuration space of a rigid body is the Lie group SE(3) - the special Euclidean group (Marsden and Scheurle 1993; Marsden and Ratiu 2003; Murray et al. 1994; Selig 2005), and the BH-equations are the Euler-Poincaré equations (when formulated in terms of body-fixed twists), or the reduced Euler-Lagrange equations (when using spatial twists). The rigid body is often used as the typical example to study fundamental aspects of nonholonomic systems in a coordinate-free geometric setting. However, a systematic treatment of the original BH-equations (Hamel 1904a, 1924, 1949) in local coordinates along with the original derivation of the Hamel coefficients for the rigid body, in a way that links them to the Euler-Lagrange equations on $S E(3)$, has not yet been reported in the literature. Key to this is evaluating the Hamel coefficients taking into account the geometry of rigid body motions (possibly without resorting to local coordinates), which not only demonstrates technicalities of the actual determination of these coefficients, but also offers insight into the geometric nature of the problem. Moreover, the Hamel coefficients are specific to the particular representation of rigid body velocities (i.e. the definition of quasi-velocities) and the corresponding invariance of the rigid body Lagrangian under action of the symmetry group. The three most relevant representations of rigid body velocities are the body-fixed, spatial, and hybrid representation (Müller 2018; Murray et al. 1994). The first two correspond to a left-invariant and right-invariant Lagrangian on $S E(3)$, respectively. The hybrid representation assumes the direct product group $S O(3) \times \mathbb{R}^{3}$ as symmetry group and corresponding right-invariant Lagrangian, which clearly implies a different geometric model of rigid body motions (Müller 2016; Müller and Terze 2014). The derivations of the Hamel coefficients for the rigid body reported in the literature almost exclusively use particular local coordinates to describe rigid body motions (see e.g. Hamel 1904a, pp. 18-20 or Papastavridis 2002, p. 369 for the rotating body, and Whittaker 1904, pp. 43 \& 217 or Lurie 2002, p. 76 for the free body), which leads to rather involved expressions. Some of the few exception are Hamel's derivation for the rotating body described in terms of the body-fixed angular velocity 
(see Hamel 1949, p. 489), and the derivation for the rotating $n$-dimensional body by Hurtado and Sinclair (2004).

In this paper, a systematic derivation of the Hamel coefficients for the rigid body and the corresponding $\mathrm{BH}$-equations are presented for the three representations of rigid body velocities mentioned above. The Hamel coefficients are derived without resorting to local coordinates (no parameterization of rigid body motions). Hamel's EulerLagrange equations equally apply to systems subjected to Pfaffian constraints, where the nonholonomic geometry is encoded in the Hamel coefficients. This is recalled and several formulations of the Hamel equations are presented. The Hamel equations for the rolling ball with inhomogenous mass density are presented, which is a typical example for a nonholonomically constrained rigid body (Holm 2011).

Despite the long history of analytical rigid body dynamics, this paper seems to be the first publication reporting an explicit geometric construction of the Hamel coefficients, which are indeed determined by the structure constant of the Lie group underlying the respective definition of rigid body twist. The paper summarizes material that is scattered in the literature along with references to the original sources, and presents novel derivations of Hamel coefficients. The paper is organized as follows. Section 2 briefly summarizes the $\mathrm{BH}$-equations in their original form and the definition of the Hamel coefficients. First the BH-equations for general unconstrained systems described in quasi-coordinates are reviewed. Then the Hamel equations for constrained systems described in quasi-coordinates as well as in 'holonomic' coordinates are recalled. In order to make a connection to the modern approach in geometric mechanics, the $\mathrm{BH}$ equations for the rigid body are finally identified as the Euler-Poincaré, respectively, the reduced Euler-Lagrange equations when using $S E(3)$ as rigid body configuration space. The Hamel coefficients are derived in Sect. 3 for the rotating rigid body when the angular velocity in body-fixed and spatial representation is used as quasi-velocity. The corresponding BH-equations are derived in Sect. 4. The Hamel coefficients for the free rigid body are derived in Sect. 5 where the body-fixed, spatial, and hybrid representations of twists are used as quasi-velocities. The corresponding BH-equations are derived explicitly in Sect. 6. In addition, the Hamel equations for an inhomogenous ball rolling on a horizontal plane are derived as an example for a nonholonomically constrained rigid body. A list of symbols and abbreviations is given in Appendix B and C, respectively. Throughout the paper, the Lagrangian will be the kinetic energy.

Notation: with reference to the original development by Hamel and Boltzmann, the classical notation in terms of local coordinates is used. Tensor notation and the Ricci summation convention is applied throughout the paper. Cartesian vectors are resolved in orthogonal frames, and their covariant coordinates (lower indices) are used. $\varepsilon_{i j k}$ denotes the Levi-Civita symbol, and $\delta_{i j}$ the Kronecker symbol.

\section{Motion Equations in Quasi-Velocities}

\subsection{Generalized Coordinates and Quasi-Velocities}

The configuration of the system is described by local coordinates $q^{a}, a=1, \ldots, n$ summarized in the vector $\mathbf{q} \in \mathbb{V}^{n}$. The system velocity is described by the 
(quasi)velocity coordinates $u^{a}, a=1, \ldots, n$, summarized in $\mathbf{u} \in \mathbb{R}^{n}$, which are related to the generalized speeds by

$$
\mathbf{u}=\mathbf{A}(\mathbf{q}) \dot{\mathbf{q}}
$$

and in components

$$
u^{a}=A_{b}^{a} \dot{q}^{b} .
$$

The transition to the corresponding (quasi)coordinates $\pi^{a}, a=1, \ldots, n$ is described by the Pfaffian forms (not to be confused with the angular velocity)

$$
\omega^{a}:=d \pi^{a}=A_{b}^{a} d q^{b}
$$

If the relation (3) is not integrable for some $a$, then the $\pi^{a}$ are referred to as quasicoordinates, and the $u^{a}$ as quasi-velocities (also called 'nonholonomic' velocities, see Remark 4).

The inverse relation to (1) is written as

$$
\dot{\mathbf{q}}=\mathbf{B}(\mathbf{q}) \mathbf{u}
$$

with $\mathbf{B}:=\mathbf{A}^{-1}$, and in components

$$
\dot{q}^{a}=B_{b}^{a} u^{b}
$$

Accordingly, the inverse of (3) is $d q^{a}=B_{b}^{a} d \pi^{b}$. In the following, the transformations are assumed to be regular.

For a given trajectory $q^{a}(t)$, the (quasi)velocities can be integrated to obtain (quasi)coordinates

$$
\pi^{a}:=\int u^{a} d t=\int A_{b}^{a} \dot{q}^{b} d t=\int \omega^{a}=\int A_{b}^{a} d q_{b} .
$$

The so obtained quantities can serve as generalized coordinates if and only if the last integral on the right-hand side is path independent. This is so if and only if $d \omega^{a} \equiv 0$ (i.e. $\omega^{a}$ are exact one-forms), and in coordinates

$$
\frac{\partial A_{b}^{a}}{\partial q^{c}}-\frac{\partial A_{c}^{a}}{\partial q^{b}} \equiv 0
$$

which are the conditions for integrability of the system of the $n$ Pfaffian forms (3).

\subsection{Rigid Body Velocities}

Rigid body motions evolve on the Lie group $S E(3)$, and the quasi-velocity is the body twist. In body-fixed representation, the latter is given by the left-invariant vector field (158) on $S E(3)$. The motion of a rigid body rotating about a fixed point evolves on 
$S O(3)$, and the quasi-velocity is the angular velocity. In body-fixed representation this is the left-invariant vector field (144) on $S O$ (3). Thus, (3) are the Maurer-Cartan forms on $S E(3)$ and $S O(3)$, respectively. They satisfy the Maurer-Cartan equations (Marsden and Ratiu 2003, p. 255)

$$
d \omega^{a}=-\frac{1}{2} c_{b c}^{a} \omega^{b} \wedge \omega^{c}
$$

which are in local coordinates

$$
\frac{\partial A_{b}^{a}}{\partial q^{c}}-\frac{\partial A_{c}^{a}}{\partial q^{b}}=c_{r s}^{a} A_{b}^{r} A_{c}^{s}
$$

where $c_{b c}^{a}$ are the structure constants of the Lie group. Clearly, the condition (7) is not satisfied for any non-Abelian group. This holds true for $S E(3)$ and $S O$ (3), asserting that rigid body velocities are non-integrable. The same argument applies to right-invariant vector fields, i.e. when using spatial representations of rigid body velocities, where the sign in (8) changes. For the Abelian motion subgroups $\mathbb{R}^{n}, S O(2), S O(2) \times \mathbb{R}$, the velocities are indeed integrable.

\subsection{The Boltzmann-Hamel Equations}

Let $L\left(q^{a}, u^{a}\right)$ be the Lagrangian in terms of (quasi)velocity coordinates $u^{a}$. For mechanical systems this is usually $L\left(q^{a}, u^{a}\right)=T\left(q^{a}, u^{a}\right)-U\left(q^{a}\right)$ with the kinetic (co)energy $T\left(q^{a}, u^{a}\right)$ and the potential energy $U\left(q^{a}\right)$, but can be a general energetic state function for a general dynamic system, e.g. given by the magnetic coenergy and electric energy for discrete electromechanical systems (Maißer et al. 1997). The variational form of the Hamel equations is (Hamel 1904a, 1924, 1949; Papastavridis 2002)

$$
\left(\frac{d}{d t} \frac{\partial L}{\partial u^{a}}-\frac{\partial L}{\partial \pi^{a}}-Q_{a}\right) \delta \pi^{a}+\frac{\partial L}{\partial u^{a}}\left(\frac{d \delta \pi^{a}-\delta d \pi^{a}}{d t}\right)=0
$$

where $Q_{a}$ are generalized forces dual to $u^{a}$. The term in the second bracket satisfies the transitory (or transpositional) relation

$$
\frac{d \delta \pi^{a}-\delta d \pi^{a}}{d t}=\gamma_{c b}^{a} u^{b} \delta \pi^{c},
$$

respectively, $d \delta \pi^{a}-\delta d \pi^{a}=\gamma_{c b}^{a} d \pi^{c} \delta \pi^{c}$, where, with the transformation (2),

$$
\gamma_{a b}^{c}:=\left(\frac{\partial A_{r}^{c}}{\partial q^{s}}-\frac{\partial A_{s}^{c}}{\partial q^{r}}\right) B_{a}^{r} B_{b}^{s}
$$

are the Hamel coefficients. The Boltzmann-Hamel equations in their explicit form are hence (Hamel 1904a, 1924, 1949; Neimark and Fufaev 1972; Papastavridis 2002)

$$
\frac{d}{d t} \frac{\partial L}{\partial u^{a}}-\frac{\partial L}{\partial \pi^{a}}+\frac{\partial L}{\partial u^{b}} \gamma_{a c}^{b} u^{c}=Q_{a} .
$$


Complemented with (5) they describe the dynamics of an unconstrained system in terms of (quasi)coordinates.

Since the Lagrangian depends on the coordinates $q^{a}$, rather than on the quasicoordinates $\pi^{a}$, for the actual evaluation of (13) the partial derivative $\frac{\partial L}{\partial \pi^{a}}$ is replaced using the Helmholtz identity $\frac{\partial q^{b}}{\partial \pi^{a}}=\frac{\partial \dot{q}^{b}}{\partial u^{a}}=B_{b}^{a}$. This yields the 'practical' form of the Hamel equations

$$
\frac{d}{d t} \frac{\partial L}{\partial u^{a}}-\frac{\partial L}{\partial q^{b}} B_{a}^{b}+\frac{\partial L}{\partial u^{b}} \gamma_{a c}^{b} u^{c}=Q_{a} .
$$

Comparing (12) and (7) shows that the Hamel coefficients vanish if and only if $u^{a}$ are integrable.

Now consider a system defined on a Lie group. With regular A, relation (9) is transformed to

$$
c_{a b}^{c}=\left(\frac{\partial A_{r}^{c}}{\partial q^{s}}-\frac{\partial A_{s}^{c}}{\partial q^{r}}\right) B_{a}^{r} B_{b}^{s} .
$$

Comparing this with (12) reveals that the Hamel coefficients are identical to the structure constants (with negative sign if spatial representation is used). This already shows that the Hamel coefficients for the rigid body are the structure constants of $S E(3)$, respectively, of $S O(3) \times \mathbb{R}^{3}$. The goal of this paper is to derive them from their original definition (12).

Remark 1 Equation (13) were published by Hamel (1904a, 1924, 1949) who referred to them as the Lagrange-Euler equations. Because Boltzmann (1902, 1904) presented a version of Lagrange's equations which is very close to Hamel's equations, (13) are usually referred to as the Boltzmann-Hamel equations (Maisser 1997; Maruskin and Bloch 2007). Further, Lurie (2002) called $\gamma_{a b}^{c}$ the Boltzmann 'three-index symbols'. In the context of parametrization of rotations, they are called Ricci coefficients.

Remark 2 The expression (12) can be reformulated as

$$
\begin{aligned}
\gamma_{a b}^{c} & =\left(B_{a}^{r} B_{b}^{s}-B_{a}^{s} B_{b}^{r}\right) \frac{\partial A_{r}^{c}}{\partial q^{s}} \\
& =A_{r}^{c}\left(\frac{\partial B_{b}^{r}}{\partial q^{a}}-\frac{\partial B_{a}^{r}}{\partial q^{b}}\right) .
\end{aligned}
$$

With regularity of $\mathbf{A}$, the last form gives rise to

$$
\frac{\partial B_{b}^{r}}{\partial q^{a}}-\frac{\partial B_{a}^{r}}{\partial q^{b}}=\gamma_{a b}^{c} B_{c}^{r} .
$$

These expressions may be advantageous for actual evaluations.

Remark 3 If $u^{a}$ are integrable, then $u^{a}=\dot{q}^{a}$ (or linear combinations of $\dot{q}^{a}$ ), and (13) reduce to the ordinary Euler-Lagrange equations on $T \mathbb{V}^{n}$

$$
\frac{d}{d t} \frac{\partial L}{\partial \dot{q}^{a}}-\frac{\partial L}{\partial q^{b}}=Q_{a}
$$


and the $q^{a}$ are called Lagrange coordinates.

\subsection{Hamel Equations for Constrained Systems}

For completeness, and since it is instructive to trace back the origin of the terminology, the Hamel equations for a system described in terms of quasi-velocities subjected to Pfaffian constraints are recalled. The naming shall reflect the fact that these equations were introduced by Hamel whereas Boltzmann exclusively considered unconstrained systems.

\subsubsection{Hamel-Equations in Terms of Quasi-Velocities}

The point of departure is the Lagrangian $L\left(q^{a}, \dot{q}^{a}\right)$ in terms of 'holonomic' coordinates. As before, $q^{a}, 1, \ldots, n$ are coordinates used to describe the configuration of the system, but now the $\dot{q}^{a}$ are subjected to a system of $\bar{m}$ Pfaffian constraints

$$
u^{\alpha}=0 \quad \text { with } \quad u^{\alpha}:=A_{a}^{\alpha} \dot{q}^{a}, \quad \alpha=1, \ldots, \bar{m}
$$

so that only $\delta:=n-\bar{m}$ of the $\dot{q}^{a}$ are independent (for simplicity catastatic constraints are considered). Thus $n-\bar{m}$ independent quasi-velocities can be introduced as

$$
u^{I}=A_{a}^{I} \dot{q}^{a}, \quad I=\bar{m}+1, \ldots, n .
$$

These independent velocity coordinates are indexed with capital Lattin letters $I, J, K, \ldots=\bar{m}+1, \ldots, n$ (w.l.o.g. the last $n-\bar{m}$ indices). The system of Pfaffian equations (20) and (21) are summarized as in (2). The solution of this system is given by (5), presuming that $\mathbf{A}$ is regular.

Denote with $\mathcal{L}\left(q^{a}, u^{a}\right):=L\left(q^{a}, B_{b}^{a} u^{b}\right)$ the Lagrangian after replacing $\dot{q}^{a}$ by $u^{a}$ according to (5). The Hamel equations of the constrained system in terms of the (quasi)velocities $u^{I}$ are Hamel (1904a, 1924, 1949)

$$
\frac{d}{d t} \frac{\partial \mathcal{L}}{\partial u^{I}}-\frac{\partial \mathcal{L}}{\partial q^{a}} B_{I}^{a}+\frac{\partial \mathcal{L}}{\partial u^{a}} \gamma_{I J}^{a} u_{J}=Q_{I}, \quad I=\bar{m}+1, \ldots, n
$$

with generalized forces $Q_{I}=B_{I}^{a} Q_{a}$, in which the $u^{\alpha}, \alpha=1, \ldots \bar{m}$ are set to zero. The terms

$$
\gamma_{I J}^{a}=\left(\frac{\partial A_{b}^{a}}{\partial q^{c}}-\frac{\partial A_{c}^{a}}{\partial q^{b}}\right) B_{I}^{b} B_{J}^{c}
$$

are the Hamel coefficients of the constrained system. The $n-\bar{m}$ Eq. (22), together with the $n$ kinematic equations

$$
\dot{q}^{a}=B_{I}^{a} u^{I}, \quad I=\bar{m}+1, \ldots, n
$$

govern the system dynamics in term of the system state $\left(q^{a}, u^{I}\right)$. 
When deriving the Hamel equations (22) for a constrained system, it is important that the Pfaffian forms $u^{\alpha}$ associated to the constraints are treated as independent just as the independent (quasi)velocities $u^{I}$. The $u^{\alpha}$ must be kept during the derivation in order to obtain the partial derivatives $\frac{\partial \mathcal{L}}{\partial u^{\alpha}}$ in (22). In the final equations, the $u^{\alpha}$ are set to zero (see example in Sect. 6.5). It is also crucial that the original 'unconstrained' Lagrangian is used in which the $\dot{q}^{a}$ are expressed in terms of all $u^{a}, a=1, \ldots, n$ using (5).

Remark 4 The constraints (20) are holonomic and the velocities $u^{I}$ are integrable if and only if

$$
\gamma_{I J}^{a} \equiv 0
$$

The fact that the constraints as well as the (quasi)velocities must satisfy equivalent integrability conditions, encoded in the coefficients (23), motivated Boltzmann (1902), and later Hamel (1904a), to call the $\pi^{a}$ 'nonholonomic coordinates' (see also Whittaker 1904 and (2002) while the quasi-velocities are called 'nonholonomic' velocities (Hamel 1949, p. 473; Sommerfeld 1948, p. 218). These terms are still used, although 'nonholonomic coordinates' seems to be an oxymoron. It should be noted that (25) is the condition for 'complete' nonholonomy. Even if (25) is not satisfied there may exist lower-dimensional integral manifolds (Bullo and Lewis 2005).

\subsubsection{Hamel-Equations in Terms of 'Holonomic Velocities'}

Consider a system described in terms of 'holonomic velocities' $\dot{q}^{a}$ subjected to $\bar{m}$ constraints. The velocities can be partitioned as $\dot{\mathbf{q}}=\left(\dot{s}^{\alpha}, \dot{r}^{I}\right)$, where $\dot{s}^{\alpha}, 1, \ldots, \bar{m}$ are dependent, and $\dot{r}^{I}, I=\bar{m}+1, \ldots, n$ are independent velocity coordinates, i.e. $u^{I}:=\dot{r}^{I}$. Together with the Pfaffian constraints (20), this can be written in matrix form as

$$
\mathbf{u}=\left(\begin{array}{cc}
\mathbf{A}_{1} & \mathbf{A}_{2} \\
\mathbf{0} & \mathbf{I}
\end{array}\right)\left(\begin{array}{l}
\dot{\mathbf{s}} \\
\dot{\mathbf{r}}
\end{array}\right)
$$

The solution of (26) is given by (4) with

$$
\mathbf{B}=\left(\begin{array}{cc}
-\mathbf{A}_{1}^{-1} & -\mathbf{A}_{1}^{-1} \mathbf{A}_{2} \\
\mathbf{0} & \mathbf{I}
\end{array}\right)
$$

With the particular form of (26), the Hamel coefficients are

$$
\gamma_{I J}^{\alpha}=\frac{\partial A_{I}^{\alpha}}{\partial r^{J}}-\frac{\partial A_{J}^{\alpha}}{\partial r^{I}}+\left(\frac{\partial A_{\beta}^{\alpha}}{\partial s^{\gamma}}-\frac{\partial A_{\gamma}^{\alpha}}{\partial s^{\beta}}\right) B_{I}^{\beta} B_{J}^{\gamma}, \quad \alpha=1, \ldots, \bar{m}
$$

and $\gamma_{I J}^{K} \equiv 0, K=\bar{m}+1, \ldots, n$.

Remark 5 It is instructive noticing that, when using velocities $\dot{q}^{a}$, the condition (25) are the original Frobenius condition (see Frobenius 1875, §13-§16)

$$
\left(\frac{\partial A_{\alpha a}}{\partial q^{b}}-\frac{\partial A_{\alpha b}}{\partial q^{a}}\right) B_{I}^{a} B_{J}^{b} \equiv 0, \quad \alpha=1, \ldots, \bar{m}
$$


for integrability of the constraints (20), and the non-vanishing coefficients in (29) are exactly the Hamel coefficients (28). The fact that $\gamma_{I J}^{K} \equiv 0$ indicates that the $\dot{q}^{a}$ are 'holonomic' velocities. If (29) holds, then (20) corresponds to a system of $m=\bar{m}$ constraints on $q$, whereas for nonholonomic constraints $m<\bar{m}$. This is why $\gamma_{I J}^{\alpha}$ was referred to as the 'object of nonholonomy' (Maisser 1997) or 'nonholonomicity' (Papastavridis 1999, 2002).

An alternative way to express the Hamel coefficients, and hence the Hamel equations, is to first solve the constraints $A_{a}^{\alpha} \dot{q}^{a}=0$ in (20). A solution in terms of the independent $\dot{r}_{I}$ is $\dot{s}^{\alpha}=-\bar{F}_{I}^{\alpha} \dot{r}_{I}, I=\bar{m}+1, \ldots, n$, where $\bar{F}_{I}^{a}$ is defined by

$$
\dot{\mathbf{q}}=\mathbf{F} \dot{\mathbf{r}}, \quad \text { with } \quad \mathbf{F}:=\left(\begin{array}{c}
-\overline{\mathbf{F}} \\
\mathbf{I}
\end{array}\right), \quad \overline{\mathbf{F}}:=\mathbf{A}_{1}^{-1} \mathbf{A}_{2} \text {. }
$$

Therewith the constraints can be rewritten as

$$
u^{\alpha}=0 \quad \text { with } \quad u^{\alpha}:=\dot{s}^{\alpha}+\bar{F}_{I}^{\alpha} \dot{r}^{I}, \quad \alpha=1, \ldots, \bar{m}
$$

and the corresponding Pfaffian system and its solution are in matrix form

$$
\mathbf{u}=\left(\begin{array}{ll}
\mathbf{I} & \overline{\mathbf{F}} \\
\mathbf{0} & \mathbf{I}
\end{array}\right) \dot{\mathbf{q}}=\overline{\mathbf{A}}(\mathbf{q}) \dot{\mathbf{q}}, \quad \dot{\mathbf{q}}=\left(\begin{array}{cc}
\mathbf{I} & -\overline{\mathbf{F}} \\
\mathbf{0} & \mathbf{I}
\end{array}\right) \mathbf{u}=\overline{\mathbf{B}}(\mathbf{q}) \mathbf{u} .
$$

The Hamel coefficients for this Pfaffian system are $\gamma_{I J}^{K} \equiv 0, K=\bar{m}+1, \ldots, n$, and

$$
\begin{aligned}
\gamma_{I J}^{\alpha} & =\frac{\partial \bar{A}_{I}^{\alpha}}{\partial r^{J}}-\frac{\partial \bar{A}_{J}^{\alpha}}{\partial r^{I}}+\left(\frac{\partial \bar{A}_{\beta}^{\alpha}}{\partial s^{\gamma}}-\frac{\partial \bar{A}_{\gamma}^{\alpha}}{\partial s^{\beta}}\right) \bar{B}_{I}^{\beta} \bar{B}_{J}^{\gamma} \\
& =\frac{\partial \bar{F}_{I}^{\alpha}}{\partial r^{J}}-\frac{\partial \bar{F}_{J}^{\alpha}}{\partial r^{I}}+\frac{\partial \bar{F}_{J}^{\alpha}}{\partial s^{\gamma}} \bar{F}_{I}^{\gamma}-\frac{\partial \bar{F}_{I}^{\alpha}}{\partial s^{\gamma}} \bar{F}_{J}^{\gamma}, \quad \alpha=1, \ldots, \bar{m}
\end{aligned}
$$

Denote with $\mathcal{L}\left(s^{\alpha}, r^{I}, u^{a}\right):=L\left(q^{a}, u^{I}\right)$ the (unconstrained) Lagrangian defined by setting $\dot{r}^{I}:=u^{I}$ and $\dot{s}^{\alpha}=u^{\alpha}-\bar{F}_{I}^{\alpha} u_{I}$ [the solution of (31)]. Noting that $\frac{\partial}{\partial u^{\alpha}}=\frac{\partial}{\partial \dot{s}^{\alpha}}$, the Hamel equations (13) of the constrained system are the Chaplygin equations

$$
\frac{d}{d t} \frac{\partial \mathcal{L}}{\partial \dot{r}^{I}}-\frac{\partial \mathcal{L}}{\partial r^{I}}+\frac{\partial \mathcal{L}}{\partial \dot{s}^{\alpha}} \gamma_{I J}^{\alpha} \dot{r}^{J}=0, \quad I=\bar{m}+1, \ldots, n .
$$

Now define the constrained Jacobian $L_{\mathrm{c}}\left(q^{a}, \dot{r}^{I}\right):=L\left(s^{\alpha}, r^{I},-\bar{F}_{I}^{\alpha} \dot{r}^{I}, \dot{r}^{I}\right)$. Since $\frac{\partial \mathcal{L}}{\partial \dot{r}^{I}}=\frac{\partial L_{\mathrm{c}}}{\partial \dot{r}^{I}}$ the Hamel equations attain the form

$$
\frac{d}{d t} \frac{\partial L_{\mathrm{c}}}{\partial \dot{r}^{I}}-\frac{\partial L_{\mathrm{c}}}{\partial r^{I}}+\frac{\partial L_{\mathrm{c}}}{\partial s^{\alpha}} \bar{F}_{I}^{\alpha}=-\frac{\partial \mathcal{L}}{\partial \dot{s}^{\alpha}} \gamma_{I J}^{\alpha} \dot{r}^{J}, \quad I=\bar{m}+1, \ldots, n
$$

which were reported in (Bloch et al. 2005), where $\bar{F}_{I}^{\alpha}$ are interpreted as (kinematic) Ehresmann connection on $T \mathbb{V}^{n}$ and $\gamma_{I J}^{\alpha}$ in (33) as its curvature. It should finally be 
remarked that Eq. (35) are the explicit form of the equations of Voronets (1901) (also spelled Woronetz 1910)

$$
\frac{d}{d t} \frac{\partial L}{\partial \dot{r}^{I}}-\frac{\partial L}{\partial r^{I}}+F_{I}^{a}\left(\frac{d}{d t} \frac{\partial L}{\partial \dot{s}^{\alpha}}-\frac{\partial L}{\partial s^{\alpha}}\right)=F_{I}^{a} Q_{a}
$$

for a system subjected to (holonomic or nonholonomic) constraints (20).

Remark 6 Denote with $F_{J}^{a}$ the components of the orthogonal complement of $\mathbf{A}$ in (30), so that $\dot{q}^{a}=F_{I}^{a} \dot{r}^{I}$. The vector fields $F_{I}:=F_{I}^{a} \frac{\partial}{\partial q^{a}}$ define the constraint distribution $D:=\operatorname{span}\left(F_{\bar{m}+1}, \ldots, F_{n}\right)$. The $\gamma_{I J}^{a}$ in (33) are the coefficients of the Lie bracket $\left[F_{I}, F_{J}\right]$ in local coordinates. Involutivity of $D$ is equivalent to the vanishing of the Hamel coefficients (33), which is necessary and sufficient for the constraints being holonomic.

Remark 7 Introduce the one-form $\omega^{\alpha}:=d s^{\alpha}+\bar{F}_{I}^{\alpha} d r^{I}, \alpha=1, \ldots, \bar{m}$ so that constraints (31) are equivalent to the Pfaffian equations $\omega^{\alpha}=0$. The exterior derivative of $\omega^{\alpha}$ is

$$
d \omega^{\alpha}=\left(\frac{\partial \bar{F}_{I}^{\alpha}}{\partial r^{J}}-\frac{\partial \bar{F}_{I}^{\alpha}}{\partial s^{\beta}} \bar{F}_{J}^{\beta}\right) d r^{J} \wedge d r^{i} .
$$

Contracting $d \omega^{\alpha}$ with $\dot{\mathbf{q}}=\left(-\bar{F}_{I}^{\alpha} \dot{r}^{I}, \dot{r}^{I}\right)$ yields $d \omega^{\alpha}(\dot{\mathbf{q}}, \cdot)=\gamma_{I J}^{\alpha} \dot{r}^{J} d r^{I}$, and thus

$$
\frac{\partial \mathcal{L}}{\partial \dot{s}^{\alpha}} \gamma_{I J}^{\alpha} \dot{r}^{J}=d \omega^{\alpha}\left(\dot{\mathbf{q}}, \frac{\partial}{\partial r^{I}}\right) .
$$

This shows again that the $\gamma_{I J}^{\alpha}$ vanish if and only if the constraints are holonomic, i.e. $d \omega^{\alpha} \equiv 0$.

\subsection{BH-Equations as Euler-Lagrange and Euler-Poincaré Equations on SE(3)}

Hamel (1904a, b) already discussed the geometric properties of the infinitesimal transformations (3) and when they form a Lie group, in which case the Hamel coefficients are identical to the structure constants of this group. In case of the rigid body, this Lie group is the special Euclidean group $S E(3)=S O(3) \ltimes \mathbb{R}^{3}$. The pose of a rigid body is represented by $C \in S E$ (3), and its twist by $\mathbf{V} \in$ se (3), see Appendix A.2. Important is the invariance of the rigid body Lagrangian under group actions, i.e. under frame transformations. The Lagrangian is left-invariant if it is independent of the choice of body-fixed frame, and it is right-invariant if it is independent from the choice of inertial frame.

Denote with $l(C, \mathbf{V})$ the rigid body Lagrangian in terms of its pose and twist, which can be considered as a trivialized version of a Lagrangian $L(C, \dot{C})$ defined on $T_{C} S E(3)$ (Marsden and Ratiu 2003; Marsden and Scheurle 1993). The BH-equations (13) are then

$$
\frac{d}{d t} \frac{\partial l}{\partial V_{i}} \pm \frac{\partial l}{\partial V_{j}} c_{i j k} V_{k}-\frac{\partial l}{\partial \pi_{i}}=W_{i}
$$


with index range $i, j, k=1, \ldots, 6$, where $\mathbf{V}, \boldsymbol{\pi} \in s e(3), \mathbf{W} \in s e^{*}(3)$ and the structure constants are given in (165). These are the Euler-Lagrange equations for a left-invariant ( + sign), respectively, right-invariant ( - sign), Lagrangian, where $\frac{\partial L}{\partial \pi_{a}}$ is the expression for $T_{I} L_{C} D_{C} l$, respectively, $T_{I} R_{C} D_{C} l$, in local coordinates, and $D_{C} l \in T^{*} G$ denotes the partial derivative of the Lagrangian $l$ w.r.t. $C \in S E$ (3). Note that, since the basis on se (3) is deduced from an orthogonal Cartesian basis, in the following, it will not be distinguished between co- and contravariant coordinates, and only subscripts will be used, e.g. $V_{i}=V^{i}$.

If $l$ does not dependent on the pose $C \in S E$ (3), then (39) become the EulerPoincaré (respectively Lie-Poisson) equations

$$
\frac{d}{d t} \frac{\partial l}{\partial V_{i}} \pm c_{i j k} \frac{\partial l}{\partial V_{j}} V_{k}=W_{i}, \quad \frac{d}{d t} \Pi_{i} \pm c_{i j k} \Pi_{j} V_{k}=W_{i}
$$

with the rigid body momentum $\Pi \in s e^{*}$ (3). They are written in vector form as (note the change of sign)

$$
\frac{d}{d t} \frac{\partial l}{\partial \mathbf{V}} \mp \mathbf{a d}_{\mathbf{V}}^{T} \frac{\partial l}{\partial \mathbf{V}}=\mathbf{W}, \quad \frac{d}{d t} \boldsymbol{\Pi} \mp \mathbf{a d}_{\mathbf{V}}^{T} \boldsymbol{\Pi}=\mathbf{W}
$$

where $\mathbf{a d}_{\mathbf{u}}$ is the matrix (163) such that $\mathbf{a d}_{\mathbf{u}} \mathbf{v}=[\mathbf{u}, \mathbf{v}]$. The actual motion is recovered solving the kinematic reconstruction Eqs. (158) and (160), respectively.

More on the equivalence of the original $\mathrm{BH}$-equations and the Euler-Poincaré equations can be found in (Neimark and Fufaev 1972; Papastavridis 2002), and in (Bloch et al. 2009). It was shown by Guen (1968), for example, that the variational equations of Volterra and the Poincaré equations (in Chetaev form) are equivalent to Hamel's variational equations, where the connection is made via the transpositional equation (11).

In the following sections, the Hamel coefficients for the rigid body are derived using local coordinates as well in a coordinate-free way noting the geometric meaning of matrix $\mathbf{A}$ in (1), respectively of $\mathbf{B}$ in (4), which maps to the Lie algebra of $S E(3)$.

\section{Hamel Coefficients for the Rotating Rigid Body}

The rotation of a rigid body is described by the rotation of a body-fixed frame $\mathcal{F}_{\mathrm{b}}$ relative to a space-fixed inertial frame (IFR) $\mathcal{F}_{0}$, which is represented by an orthogonal matrix $\mathbf{R}=\left(R_{i j}\right) \in S O$ (3). This rotation matrix transforms coordinates of a vector resolved in $\mathcal{F}_{\mathrm{b}}$ to its coordinates when resolved in $\mathcal{F}_{0}$, i.e. it transforms body-fixed to inertial coordinates.

The Hamel coefficients are indeed specific to a particular representation of the angular velocity. In the following, the angular velocity of the body-fixed frame $\mathcal{F}_{\mathrm{b}}$ relative to the IFR $\mathcal{F}_{0}$ represented in the body-fixed frame (body-fixed representation $\omega^{\mathrm{b}}$ ) and in the IFR (spatial representation $\omega^{\mathrm{s}}$ ) are considered, as defined in (145) and (153), respectively. For both representations, the Hamel coefficients will be derived. The explicit definition (12) and the transitory relations are used to illustrate the applica- 
tion of either method. Rotation matrices are canonically parametrized in terms of three independent variables (rotation angles, axis-angle) serving as generalized coordinates. Indices $i, j, k, l=1,2,3$ refer to the Cartesian basis, indices $a, b, c=1,2,3$ refer to the generalized coordinates used to describe rotations. Since vectors are resolved in orthogonal Cartesian bases, it will not be distinguished between co- and contravariant coordinates, and the corresponding indices are written as subscripts.

\subsection{Derivation from the Explicit Definition}

The angular velocity serves as quasi-velocity. The Hamel coefficients are now derived from the explicit definition (12) for the body-fixed representation of angular velocity. For spatial rotations, the matrices $\mathbf{A}$ and $\mathbf{B}$ are placed by $\boldsymbol{\Omega}$ in (145) and $\boldsymbol{\Psi}$ in (151), respectively (superscript $\mathrm{b}$ will be omitted). Thus (12) becomes

$$
\gamma_{i j}^{k}=\left(\frac{\partial \Omega_{k a}}{\partial \varphi^{b}}-\frac{\partial \Omega_{k b}}{\partial \varphi^{a}}\right) \Psi_{i}^{a} \Psi_{j}^{b}, \quad i, j, k=1,2,3 .
$$

For fixed $k$, a skew symmetric tensor can be associated to $\gamma_{i j}^{k}$, which is expressed with (149) as

$$
\varepsilon_{r k s} \gamma_{i j}^{k}=\varepsilon_{r k s}\left(\frac{\partial \Omega_{k a}}{\partial \varphi^{b}}-\frac{\partial \Omega_{k b}}{\partial \varphi^{a}}\right) \Psi_{i}^{a} \Psi_{j}^{b}=\left(\frac{\partial^{2} \omega_{r s}}{\partial \varphi^{b} \partial \dot{\varphi}^{a}}-\frac{\partial^{2} \omega_{r s}}{\partial \dot{\varphi}^{a} \partial \varphi^{b}}\right) \Psi_{i}^{a} \Psi_{j}^{b} .
$$

Using (146), the partial derivatives of the first term in the bracket of the last term is

$$
\frac{\partial^{2} \omega_{r s}}{\partial \varphi^{b} \partial \dot{\varphi}^{a}}=R_{l r} \frac{\partial^{2} R_{l s}}{\partial \varphi^{a} \partial \varphi^{b}}+\frac{\partial R_{l r}}{\partial \varphi^{b}} \frac{\partial R_{l s}}{\partial \varphi^{a}}
$$

and analogously for the second term by swapping the indices $b$ and $a$, and thus

$$
\varepsilon_{r k s} \gamma_{i j}^{k}=\left(\frac{\partial R_{l r}}{\partial \varphi^{b}} \frac{\partial R_{l s}}{\partial \varphi^{a}}-\frac{\partial R_{l r}}{\partial \varphi^{a}} \frac{\partial R_{l s}}{\partial \varphi^{b}}\right) \Psi_{i}^{a} \Psi_{j}^{b}
$$

Replacing the partial derivatives with the relation (152) yields

$$
\varepsilon_{r k s} \gamma_{i j}^{k}=R_{l k} \varepsilon_{k j r} R_{l p} \varepsilon_{p i s}-R_{l p} \varepsilon_{p i r} R_{l k} \varepsilon_{k j s}=\varepsilon_{k j r} \varepsilon_{k i s}-\varepsilon_{k i r} \varepsilon_{k j s}=\varepsilon_{r k s} \varepsilon_{k i j}
$$

where the last term follows with $\varepsilon_{k i s} \varepsilon_{k j r}-\varepsilon_{k i r} \varepsilon_{k j s}=\delta_{i j} \delta_{r s}-\delta_{i r} \delta_{j s}-\delta_{i j} \delta_{r s}+\delta_{i s} \delta_{j r}=$ $\delta_{i s} \delta_{j r}-\delta_{i r} \delta_{j s}=\varepsilon_{k s r} \varepsilon_{k i j}=\varepsilon_{r p s} \varepsilon_{p i j}$. The Hamel coefficients for the rotating rigid body in terms of body-fixed angular velocity are thus

$$
\gamma_{i j}^{k}=\varepsilon_{k i j}
$$

An analog derivation for the spatial representation yields the Hamel coefficients $\gamma_{i j}^{k}=$ $-\varepsilon_{k i j}$. 


\subsection{Derivation via the Transitory Relation}

The Hamel coefficients can be found directly from the transitory relation (11), instead of explicitly evaluating the definition (12) in terms of local coordinates on $S O$ (3). It will turn out that this is a straightforward and simple approach. Hamel already pointed out that it might be easier to derive the Euler-Lagrange equations by directly evaluating the transitory relations. He used vectorial relations for derivatives of the angular velocity, and avoided explicit coordinate expressions (see Hamel 1949, p. 488 and also the discussion in Papastavridis 2002, p. 374).

\subsubsection{Angular Velocity in Body-Fixed Representation}

Denote with $\boldsymbol{\theta}^{\mathrm{b}} \in \mathbb{R}^{3}$ the vector of quasi-coordinates associated to the rotational motion according to $d \boldsymbol{\theta}^{\mathrm{b}}=\boldsymbol{\omega}^{\mathrm{b}} d t$, which can be regarded as 'quasi-rotation vector' represented in the moving body-fixed frame (but it is not a vector along the instantaneous rotation axis). The differential of these quasi-coordinates can be expressed using the definition (144) of the body-fixed angular velocity as

$$
d \widetilde{\boldsymbol{\theta}}^{\mathrm{b}}=\widetilde{\boldsymbol{\omega}}^{\mathrm{b}} d t=\mathbf{R}^{T} \frac{\partial \mathbf{R}}{\partial \varphi^{a}} d \varphi^{a}=\mathbf{R}^{T} d \mathbf{R}
$$

and analogously the variation as $\delta \widetilde{\boldsymbol{\theta}}^{\mathrm{b}}=\mathbf{R}^{T} \delta \mathbf{R}$. The subscript b will be omitted in the following. In case of spatial rotations, the transitory relation (11) is

$$
\frac{d}{d t}\left(d \delta \theta_{k}-\delta d \theta_{k}\right)=\gamma_{i j}^{k} \omega_{j} \delta \theta_{i}
$$

The bracketed term on the left reads in vector form $d \delta \boldsymbol{\theta}-\delta d \boldsymbol{\theta}$. Writing the latter as skew symmetric matrix, and using (48) yields

$$
\begin{aligned}
d \delta \widetilde{\boldsymbol{\theta}}-\delta d \widetilde{\boldsymbol{\theta}} & =d\left(\mathbf{R}^{T} \delta \mathbf{R}\right)-\delta\left(\mathbf{R}^{T} d \mathbf{R}\right) \\
& =d \mathbf{R}^{T} \delta \mathbf{R}+\mathbf{R}^{T} d \delta \mathbf{R}-\delta \mathbf{R}^{T} d \mathbf{R}-\mathbf{R}^{T} \delta d \mathbf{R} \\
& =-\mathbf{R}^{T} d \mathbf{R} \mathbf{R}^{T} \delta \mathbf{R}+\mathbf{R}^{T} d \delta \mathbf{R}+\mathbf{R}^{T} \delta \mathbf{R R}^{T} d \mathbf{R}-\mathbf{R}^{T} \delta d \mathbf{R} \\
& =\delta \widetilde{\boldsymbol{\theta}} d \widetilde{\boldsymbol{\theta}}-d \widetilde{\boldsymbol{\theta}} \delta \widetilde{\boldsymbol{\theta}}+\mathbf{R}^{T}(d \delta \mathbf{R}-\delta d \mathbf{R}) .
\end{aligned}
$$

Noting that the term $d \delta \mathbf{R}-\delta d \mathbf{R}$ vanishes identically, this simplifies to

$$
d \delta \widetilde{\boldsymbol{\theta}}-\delta d \widetilde{\boldsymbol{\theta}}=\delta \widetilde{\boldsymbol{\theta}} d \widetilde{\boldsymbol{\theta}}-d \widetilde{\boldsymbol{\theta}} \delta \tilde{\boldsymbol{\theta}}=[\delta \widetilde{\boldsymbol{\theta}}, d \widetilde{\boldsymbol{\theta}}]
$$

Transforming (51) to vector form, using (143), and using the vector form of the Lie bracket (155), yields $(d \delta \boldsymbol{\theta}-\delta d \boldsymbol{\theta})=\mathbf{a d}_{\delta \boldsymbol{\theta}} d \boldsymbol{\theta}=\delta \widetilde{\boldsymbol{\theta}} d \boldsymbol{\theta}$. The vector form of the transitory relation (49) follows with $\boldsymbol{\omega}=\frac{d \boldsymbol{\theta}}{d t}$ as $\frac{d}{d t}(d \delta \boldsymbol{\theta}-\delta d \boldsymbol{\theta})=\mathbf{a d}_{\delta \boldsymbol{\theta}} \boldsymbol{\omega}$. With (156), the components of this relation are

$$
\frac{d}{d t}\left(d \delta \theta_{k}-\delta d \theta_{k}\right)=\varepsilon_{k i j} \delta \theta_{i} \omega_{j}
$$


Comparing (52) and (49) confirms that $\gamma_{i j}^{k}=\varepsilon_{k i j}$, in accordance to (47). Clearly, the derivation of the Hamel coefficients via the transitory relations is simpler than using the explicit definition (12).

\subsubsection{Angular Velocity in Spatial Representation}

The vector $\boldsymbol{\theta}^{\mathrm{s}}$ of quasi-coordinates ('quasi-rotation vector'), represented in IFR, is defined by $d \theta^{\mathrm{s}}=\omega^{\mathrm{s}} d t$ with spatial representation of the angular velocity (153). It thus satisfies the relations $d \widetilde{\boldsymbol{\theta}}^{\mathrm{s}}=d \mathbf{R} \mathbf{R}^{T}$ and $\delta \widetilde{\boldsymbol{\theta}}^{\mathrm{s}}=\delta \mathbf{R} \mathbf{R}^{T}$. The transitory relation (49) applies in terms of $\boldsymbol{\theta}^{\mathrm{s}}$, and the derivation of the Hamel coefficients proceeds as above. The subscript $\mathrm{s}$ will again be omitted. Noting again that $d \delta \mathbf{R}-\delta d \mathbf{R} \equiv \mathbf{0}$, yields

$$
\begin{aligned}
d \delta \widetilde{\boldsymbol{\theta}}-\delta d \widetilde{\boldsymbol{\theta}} & =d\left(\delta \mathbf{R} \mathbf{R}^{T}\right)-\delta\left(d \mathbf{R} \mathbf{R}^{T}\right) \\
& =d \delta \mathbf{R} \mathbf{R}^{T}-\delta \mathbf{R} \mathbf{R}^{T} d \mathbf{R} \mathbf{R}^{T}-\delta d \mathbf{R} \mathbf{R}^{T}+d \mathbf{R} \mathbf{R}^{T} \delta \mathbf{R R}^{T} \\
& =(d \delta \mathbf{R}-\delta d \mathbf{R}) \mathbf{R}^{T}+d \tilde{\boldsymbol{\theta}} \delta \tilde{\boldsymbol{\theta}}-\delta \tilde{\boldsymbol{\theta}} d \tilde{\boldsymbol{\theta}} \\
& =[d \widetilde{\boldsymbol{\theta}} \delta \tilde{\boldsymbol{\theta}}],
\end{aligned}
$$

which is equivalent to the vector form $\frac{d}{d t}(d \delta \boldsymbol{\theta}-\delta d \boldsymbol{\theta})=-\mathbf{a d}_{\delta \pi} \boldsymbol{\omega}$. The coordinate form (156) of the Lie bracket yields

$$
\frac{d}{d t}\left(d \delta \theta_{k}-\delta d \theta_{k}\right)=-\varepsilon_{k i j} \delta \theta_{i} \omega_{j} .
$$

Comparing (54) with (49) in terms of the spatial representation shows that the Hamel coefficients are

$$
\gamma_{i j}^{k}=-\varepsilon_{k i j},
$$

which have the opposite sign as those when using the angular velocity in body-fixed representation.

\section{Boltzmann-Hamel Equations for Rigid Body with Fixed Point}

\subsection{Body-Fixed Angular Velocity}

For simplicity, no potential forces are included, so that the Lagrangian is identical to the kinetic energy. Denote with $\Theta^{\mathrm{b}}$ the constant inertia tensor w.r.t. the center of rotation represented in the body-fixed frame $\mathcal{F}_{\mathrm{b}}$. The kinetic energy of the rotating body in terms of the angular velocity in body-fixed representation is

$$
T\left(\omega^{\mathrm{b}}\right)=\frac{1}{2} \omega_{i}^{\mathrm{b}} \Theta_{i j}^{\mathrm{b}} \omega_{j}^{\mathrm{b}}=\frac{1}{2} \omega^{\mathrm{b} T} \Theta^{\mathrm{b}} \omega^{\mathrm{b}} .
$$

Since $T$ does not depend on the orientation, the BH-equations (13) simplify to

$$
\frac{d}{d t} \frac{\partial T}{\partial \omega_{i}^{\mathrm{b}}}+\frac{\partial T}{\partial \omega_{j}^{\mathrm{b}}} \gamma_{i k}^{j} \omega_{k}^{\mathrm{b}}=\tau_{i}^{\mathrm{b}}
$$


where $\tau_{i}^{\mathrm{b}}$ are components of the applied torque resolved in $\mathcal{F}_{\mathrm{b}}$. With $\frac{\partial T}{\partial \omega_{i}^{\mathrm{b}}}=\Theta_{i j}^{\mathrm{b}} \omega_{j}^{\mathrm{b}}$ and with the Hamel coefficients $\gamma_{i k}^{j}=\varepsilon_{j i k}=\varepsilon_{i k j}$ in (47), the $\mathrm{BH}$-equations are

$$
\Theta_{i j}^{\mathrm{b}} \dot{\omega}_{j}^{\mathrm{b}}+\varepsilon_{i k j} \omega_{k}^{\mathrm{b}} \Theta_{j l}^{\mathrm{b}} \omega_{l}^{\mathrm{b}}=\tau_{i}^{\mathrm{b}}
$$

These are indeed the dynamic Euler-equations for a rotating rigid body with fixed point in terms of body-fixed angular velocity, which are the Euler-Poincaré equations (41) on $S O(3)$, with the structure constants $\gamma_{i j}^{k}=\varepsilon_{k i j}$, for the left-invariant kinetic energy (56). They attain the well-known matrix form

$$
\Theta^{\mathrm{b}} \dot{\omega}^{\mathrm{b}}+\widetilde{\omega}^{\mathrm{b}} \Theta^{\mathrm{b}} \omega^{\mathrm{b}}=\boldsymbol{\emptyset}^{\mathrm{b}}
$$

\subsection{Spatial Angular Velocity}

The kinetic energy of the rotating body is

$$
T\left(\boldsymbol{\varphi}, \omega^{\mathrm{s}}\right)=\frac{1}{2} \omega_{i}^{\mathrm{s}} \Theta_{i j}^{\mathrm{s}} \omega_{j}^{\mathrm{s}}=\frac{1}{2} \omega^{\mathrm{s} T} \boldsymbol{\Theta}^{\mathrm{s}} \omega^{\mathrm{s}}
$$

where $\Theta^{\mathrm{s}}(\boldsymbol{\varphi})$ is the inertia tensor w.r.t. to the fixed point expressed in the IFR, and $\varphi^{a}$ are local coordinates on $S O(3)$. The spatial inertia tensor depends on the orientation and is related to the (constant) body-fixed inertia tensor as $\boldsymbol{\Theta}^{\mathrm{s}}=\mathbf{R} \boldsymbol{\Theta}^{\mathrm{b}} \mathbf{R}^{T}$.

The BH-equations (14) in terms of spatial angular velocity, and with the Hamel coefficients $\gamma_{i k}^{j}=-\varepsilon_{j i k}$ in (55), are

$$
\frac{d}{d t} \frac{\partial T}{\partial \omega_{i}^{\mathrm{s}}}-\frac{\partial T}{\partial \varphi^{a}} \Psi_{i}^{a, \mathrm{~s}}+\frac{\partial T}{\partial \omega_{j}^{\mathrm{s}}} \gamma_{i k}^{j} \omega_{k}^{\mathrm{s}}=\tau_{i}^{\mathrm{s}}
$$

where $\tau_{i}$ are the components of the applied torque, and $\Psi_{j}^{a, \mathrm{~s}}$ is the inverse of $\Omega_{i a}^{\mathrm{s}}$ in (153).

From $\frac{\partial T}{\partial \omega_{i}^{\mathrm{s}}}=\Theta_{i j}^{\mathrm{s}} \omega_{j}^{\mathrm{s}}$, and using the relation $\dot{\Theta}^{\mathrm{s}}=\dot{\mathbf{R}} \Theta^{\mathrm{b}} \mathbf{R}^{T}-\mathbf{R} \Theta^{\mathrm{b}} \mathbf{R}^{T} \dot{\mathbf{R}} \mathbf{R}^{T}=$ $\widetilde{\omega}^{\mathrm{s}} \boldsymbol{\Theta}^{\mathrm{s}}-\boldsymbol{\Theta}^{\mathrm{s}} \widetilde{\boldsymbol{\omega}}^{\mathrm{s}}$, yields

$$
\frac{d}{d t} \frac{\partial T}{\partial \omega_{i}^{\mathrm{s}}}=\Theta_{i j}^{\mathrm{s}} \dot{\omega}_{j}^{\mathrm{s}}+\dot{\Theta}_{i j} \omega_{j}^{\mathrm{s}}=\Theta_{i j}^{\mathrm{s}} \dot{\omega}_{j}^{\mathrm{s}}+\varepsilon_{i k l} \omega_{k}^{\mathrm{s}} \Theta_{l j}^{\mathrm{s}} \omega_{j}^{\mathrm{s}} .
$$

The partial derivative of $\boldsymbol{\Theta}^{\mathrm{s}}$ is found with (153) as 


$$
\begin{aligned}
\frac{\partial \boldsymbol{\Theta}^{\mathrm{s}}}{\partial \varphi^{a}} & =\frac{\partial \mathbf{R}}{\partial \varphi^{a}} \boldsymbol{\Theta}^{\mathrm{b}} \mathbf{R}^{T}-\mathbf{R} \boldsymbol{\Theta}^{\mathrm{b}} \mathbf{R}^{T} \frac{\partial \mathbf{R}}{\partial \varphi^{a}} \mathbf{R}^{T}=\widetilde{\boldsymbol{\Omega}}_{a}^{\mathrm{s}} \mathbf{R} \boldsymbol{\Theta}^{\mathrm{b}} \mathbf{R}^{T}-\mathbf{R} \boldsymbol{\Theta}^{\mathrm{b}} \mathbf{R}^{T} \widetilde{\boldsymbol{\Omega}}_{a}^{\mathrm{s}} \\
& =\widetilde{\boldsymbol{\Omega}}_{a}^{\mathrm{s}} \boldsymbol{\Theta}^{\mathrm{s}}-\boldsymbol{\Theta}^{\mathrm{s}} \widetilde{\boldsymbol{\Omega}}_{a}^{\mathrm{s}} .
\end{aligned}
$$

This gives rise to the partial derivatives of the kinetic energy (omitting superscript s)

$$
\begin{aligned}
\frac{\partial T}{\partial \varphi^{a}} & =\frac{1}{2} \omega_{j} \frac{\partial \Theta_{j k}}{\partial \varphi^{a}} \omega_{k}=\frac{1}{2}\left(\omega_{j} \varepsilon_{j l s} \Omega_{l a} \Theta_{s k} \omega_{k}-\omega_{j} \Theta_{j s} \varepsilon_{s l k} \Omega_{l a} \omega_{k}\right) \\
& =-\Omega_{j a} \varepsilon_{j s l} \omega_{s} \Theta_{l k} \omega_{k} .
\end{aligned}
$$

Finally, inserting (63) and (64) into (62) yields

$$
\Theta_{i j} \dot{\omega}_{j}+\varepsilon_{i k l} \omega_{k} \Theta_{l j} \omega_{j}+\Psi_{i}^{a} \Omega_{k a} \varepsilon_{k s l} \omega_{s} \Theta_{l r} \omega_{r}-\varepsilon_{j i k} \Theta_{j s} \omega_{s} \omega_{k}=\tau_{i}
$$

Noting that $\Psi_{i}^{a} \Omega_{k a}=\delta_{i k}$ and $\varepsilon_{i k l} \omega_{k} \Theta_{l j} \omega_{j}=\varepsilon_{j i k} \Theta_{j s} \omega_{s} \omega_{k}$, the dynamic Eulerequations for the rigid body rotating about a fixed point in terms of the spatial angular velocity are

$$
\Theta_{i j}^{\mathrm{s}} \dot{\omega}_{j}^{\mathrm{s}}+\varepsilon_{i k l} \omega_{k}^{\mathrm{s}} \Theta_{l j}^{\mathrm{s}} \omega_{j}^{\mathrm{s}}=\tau_{i}^{\mathrm{s}} .
$$

These are the Euler-Lagrange equations (39) w.r.t. the right-invariant kinetic energy (60).

Equations. (66) and (58) are structurally identical, but clearly their derivation from the kinetic energy is different. Moreover, (66) can be derived from spatial momentum conservation as

$$
\dot{L}_{j}^{\mathrm{s}}=\tau_{i}^{\mathrm{s}}
$$

where $L_{i}:=\Theta_{i j}^{\mathrm{s}} \omega_{j}^{\mathrm{s}}$ is the spatial angular momentum.

\section{Hamel Coefficients for the Spatially Moving Rigid Body}

\subsection{Derivation from the Explicit Definition}

For sake of brevity, in the following only the body-fixed representation of rigid body twists is considered, as defined in (158). The derivation for the spatial representation proceeds analogously.

The derivation proceeds as for the rotating rigid body in Sect. 3.1. The superscript $\mathrm{b}$ of $V^{\mathrm{b}}$ indicating body-fixed representation will be omitted. The Hamel coefficients $\gamma_{i j}^{k}, i, j, k=1, \ldots, 6$ are written in form of an $s e(3)$-tensor $h_{\alpha k \beta} \gamma_{i j}^{k}, \alpha, \beta=1, \ldots, 4$. Inserting the relation (169) for the body-fixed twist in the definition (12), and using (170) and (172) yields

$$
\begin{aligned}
h_{\alpha k \beta} \gamma_{i j}^{k} & =h_{\alpha i \beta}\left(\frac{\partial J_{k a}}{\partial q^{b}}-\frac{\partial J_{k b}}{\partial q^{a}}\right) G_{i}^{a} G_{j}^{b} \\
& =\left(\frac{\partial^{2} V_{\alpha \beta}}{\partial q^{b} \partial \dot{q}^{a}}-\frac{\partial^{2} V_{\alpha \beta}}{\partial q^{a} \partial \dot{q}^{b}}\right) G_{i}^{a} G_{j}^{b} .
\end{aligned}
$$


With (170), the mixed partial derivatives of the twist tensor are

$$
\frac{\partial^{2} V_{\alpha \beta}}{\partial q^{b} \partial \dot{q}^{a}}=D_{\alpha \sigma} \frac{\partial^{2} C_{\sigma \beta}}{\partial q^{b} \partial q^{a}}+\frac{\partial D_{\alpha \sigma}}{\partial q^{b}} \frac{\partial C_{\sigma \beta}}{\partial q^{a}} .
$$

Noting that the second-order derivatives commute, this leads to

$$
h_{\alpha k \beta} \gamma_{i j}^{k}=\left(\frac{\partial D_{\alpha \sigma}}{\partial q^{b}} \frac{\partial C_{\sigma \beta}}{\partial q^{a}}-\frac{\partial D_{\alpha \sigma}}{\partial q^{a}} \frac{\partial C_{\sigma \beta}}{\partial q^{b}}\right) G_{i}^{a} G_{j}^{b}
$$

Inserting (174) into (71), and noting that $D_{\mu \sigma} C_{\sigma \nu}=\delta_{\mu \nu}$, yields

$$
\begin{aligned}
h_{\alpha i \beta} \gamma_{i j}^{k} & =h_{\alpha j \mu} D_{\mu \sigma} C_{\sigma \nu} h_{v i \beta}-h_{\alpha i \mu} D_{\mu \sigma} C_{\sigma \nu} h_{v j \beta} \\
& =h_{\alpha j v} h_{v i \beta}-h_{\alpha i v} h_{v j \beta} \\
& =h_{\alpha k \beta} c_{k i j}
\end{aligned}
$$

where the identity (166) is used. The Hamel coefficients $\gamma_{i j}^{k}=c_{k i j}$, for the spatially moving rigid body are thus the structure constants (165) of $S E(3)$, when the body-fixed twist is used as quasi-velocity.

\subsection{Derivation via the Transitory Relation}

\subsubsection{Body-Fixed Representation of Rigid Body Twists}

Instead of explicitly evaluating the definition (12), the Hamel coefficients are derived from the transitory relation (11), as in sec. 3.2. Denote with $\pi^{\mathrm{b}}=\left(\boldsymbol{\theta}^{\mathrm{b}}, \boldsymbol{\rho}^{\mathrm{b}}\right) \in \mathbb{R}^{6}$ the vector of quasi-coordinates associated to the rigid body motion so that $d \pi^{\mathrm{b}}=\mathbf{V}^{\mathrm{b}} d t$, which can be regarded as 'quasi-screw coordinates' expressed in the body-fixed frame. With the definition (158) of the body-fixed twist, the differential and variation of these quasi-coordinates are expressed as

$$
\begin{aligned}
& \delta \widehat{\boldsymbol{\pi}}^{\mathrm{b}}=\mathbf{C}^{-1} \delta \mathbf{C}=\left(\begin{array}{cc}
\mathbf{R}^{T} \delta \mathbf{R} \mathbf{R}^{T} \delta \mathbf{r} \\
\mathbf{0} & 0
\end{array}\right)=\left(\begin{array}{cc}
\delta \widetilde{\boldsymbol{\theta}}^{\mathrm{b}} & \delta \boldsymbol{\rho}^{\mathrm{b}} \\
\mathbf{0} & 0
\end{array}\right), d \widehat{\boldsymbol{\pi}}^{\mathrm{b}} \\
& =\mathbf{C}^{-1} d \mathbf{C}=\left(\begin{array}{cc}
\mathbf{R}^{T} d \mathbf{R} \mathbf{R}^{T} d \mathbf{r} \\
\mathbf{0} & 0
\end{array}\right) .
\end{aligned}
$$

The term $d \widetilde{\boldsymbol{\theta}}^{\mathrm{b}}=\mathbf{R}^{T} d \mathbf{R}$ defines the differential $d \boldsymbol{\theta}^{\mathrm{b}}$ of the 'quasi-rotation vector' in (48), and $d \rho^{\mathrm{b}}=\mathbf{R}^{T} d \mathbf{r}$ is the differential of the position vector of the body-fixed frame expressed in the body-fixed frame. Again, in the following, the subscript $b$ will be omitted.

Written in vector form, the transitory relation (11) involves the term $d \delta \boldsymbol{\pi}-\delta d \boldsymbol{\pi}$, which is equivalent to the matrix form $d \delta \widehat{\boldsymbol{\pi}}-\delta d \widehat{\boldsymbol{\pi}}$ using the isomorphism (159). 
Inserting (73) yields

$$
\begin{aligned}
d \delta \widehat{\boldsymbol{\pi}}-\delta d \widehat{\boldsymbol{\pi}} & =d\left(\mathbf{C}^{-1} \delta \mathbf{C}\right)-\delta\left(\mathbf{C}^{-1} d \mathbf{C}\right) \\
& =d \mathbf{C}^{-1} \delta \mathbf{C}+\mathbf{C}^{-1} d \delta \mathbf{C}-\delta \mathbf{C}^{-1} d \mathbf{C}-\mathbf{C}^{-1} \delta d \mathbf{C} \\
& =-\mathbf{C}^{-1} d \mathbf{C} \mathbf{C}^{-1} \delta \mathbf{C}+\mathbf{C}^{-1} d \delta \mathbf{C}+\mathbf{C}^{-1} \delta \mathbf{C C}^{-1} d \mathbf{C}-\mathbf{C}^{-1} \delta d \mathbf{C} \\
& =\delta \widehat{\boldsymbol{\pi}} d \widehat{\boldsymbol{\pi}}-d \widehat{\boldsymbol{\pi}} \delta \widehat{\boldsymbol{\pi}}+\mathbf{C}^{-1}(d \delta \mathbf{C}-\delta d \mathbf{C}) \\
& =\delta \widehat{\boldsymbol{\pi}} d \widehat{\boldsymbol{\pi}}-d \widehat{\boldsymbol{\pi}} \delta \widehat{\boldsymbol{\pi}}=[\delta \widehat{\boldsymbol{\pi}}, d \widehat{\boldsymbol{\pi}}] .
\end{aligned}
$$

The Lie bracket, and thus (74), can be written in vector form using (163). Making use of the skew symmetry of the Lie bracket, (74) is equivalent to $d \delta \boldsymbol{\pi}-\delta d \boldsymbol{\pi}=-\operatorname{ad}_{d \boldsymbol{\pi}} \delta \boldsymbol{\pi}$. The transitory relation (11) thus becomes, with $d \boldsymbol{\pi}=\mathbf{V} d t$,

$$
\frac{d}{d t}(d \delta \boldsymbol{\pi}-\delta d \boldsymbol{\pi})=-\mathbf{a d} \mathbf{v} \delta \boldsymbol{\pi}=-\left(\begin{array}{c}
\widetilde{\boldsymbol{\omega}} \delta \boldsymbol{\theta} \\
\widetilde{\boldsymbol{\omega}} \delta \boldsymbol{\rho}+\widetilde{\mathbf{v}} \delta \boldsymbol{\theta}
\end{array}\right)
$$

In components, $(75)$ reads

$$
\begin{aligned}
\frac{d}{d t}\left(d \delta \pi_{k}-\delta d \pi_{k}\right) & =-c_{k i j} V_{i} \delta \pi_{j} \\
& =c_{k i j} \delta \pi_{i} V_{j}
\end{aligned}
$$

Comparing (77) and (11) shows again that the Hamel coefficients $\gamma_{i j}^{k}=c_{k i j}$ are the structure constants of $S E(3)$. The above derivation is much simpler than using the definition of Hamel coefficients (12) as was already mentioned by Hamel. Moreover, the above matrix representation of $S E(3)$ further simplifies the derivation.

\subsubsection{Spatial Representation of Rigid Body Twists}

The vector of quasi-coordinates $\pi^{\mathrm{s}}$ ('quasi-screw coordinates' represented in IFR) is introduced so that $d \boldsymbol{\pi}^{\mathrm{s}}=\mathbf{V}^{\mathrm{s}} d t$. It is determined by the expression (160) for the rigid body twist in spatial representation as

$$
\begin{aligned}
\delta \widehat{\boldsymbol{\pi}}^{\mathrm{s}} & =\delta \mathbf{C} \mathbf{C}^{-1}=\left(\begin{array}{cc}
\delta \mathbf{R} \mathbf{R}^{T} & \delta \mathbf{r}-\delta \tilde{\boldsymbol{\theta}} \mathbf{r} \\
\mathbf{0} & 0
\end{array}\right)=\left(\begin{array}{cc}
\delta \widetilde{\boldsymbol{\theta}}^{\mathrm{s}} & \delta \boldsymbol{\rho}^{\mathrm{s}} \\
\mathbf{0} & 0
\end{array}\right), \quad d \widehat{\boldsymbol{\pi}}^{\mathrm{s}}=d \mathbf{C} \mathbf{C}^{-1} \\
& =\left(\begin{array}{cc}
d \mathbf{R} \mathbf{R}^{T} & d \mathbf{r} \mathbf{R}^{T} \\
\mathbf{0} & 0
\end{array}\right)
\end{aligned}
$$

where $\delta \widetilde{\boldsymbol{\theta}}^{\mathrm{s}}=\delta \mathbf{R} \mathbf{R}^{T}$ defines the variation $\delta \boldsymbol{\theta}^{\mathrm{s}}$ of the 'quasi-rotation vector' (Sect. 3.2.2), and $\delta \rho^{s}=\delta \mathbf{r}-\delta \tilde{\boldsymbol{\theta}} \mathbf{r}$ is the variation of the position vector of that point attached to the body which momentarily coincides with the origin of the IFR. Both vectors are represented in the IFR. Noting that $d \delta \mathbf{C}-\delta d \mathbf{C} \equiv \mathbf{0}$, yields (omitting the subscript s) 


$$
\begin{aligned}
d \delta \widehat{\boldsymbol{\pi}}-\delta d \widehat{\boldsymbol{\pi}} & =d\left(\delta \mathbf{C} \mathbf{C}^{-1}\right)-\delta\left(d \mathbf{C} \mathbf{C}^{-1}\right) \\
& =d \delta \mathbf{C} \mathbf{C}^{-1}-\delta \mathbf{C} \mathbf{C}^{-1} d \mathbf{C} \mathbf{C}^{-1}-\delta d C \mathbf{C}^{-1}+d \mathbf{C} \mathbf{C}^{-1} \delta \mathbf{C} \mathbf{C}^{-1} \\
& =(d \delta \mathbf{C}-\delta d \mathbf{C}) \mathbf{C}^{-1}+d \widehat{\boldsymbol{\pi}} \delta \widehat{\boldsymbol{\pi}}-\delta \widehat{\boldsymbol{\pi}} d \widehat{\boldsymbol{\pi}} \\
& =[d \widehat{\boldsymbol{\pi}}, \delta \widehat{\boldsymbol{\pi}}] .
\end{aligned}
$$

Inserting this in the transitory relation (11), and using the vector form (162) of the Lie bracket leads to

$$
\frac{d}{d t}(d \delta \boldsymbol{\pi}-\delta d \boldsymbol{\pi})=\operatorname{ad}_{\mathbf{V}} \delta \boldsymbol{\pi} .
$$

The coordinate expression (164) of the Lie bracket yields, with $d \boldsymbol{\pi} / d t=\mathbf{V}$,

$$
\frac{d}{d t}\left(d \delta \pi_{k}-\delta d \pi_{k}\right)=-c_{k i j} \delta \pi_{i} V_{j}
$$

Comparing (81) with (11) shows that the Hamel coefficients are

$$
\gamma_{i j}^{k}=-c_{k i j}
$$

which are identical to those in (77) when using the the body-fixed representation of twists, but with opposite sign.

\subsubsection{Hybrid Representation of Rigid Body Twists}

A representation of twists frequently found in the literature, in particular for computational multibody dynamics, is the so-called hybrid representation (Murray et al. 1994). The twist vector is denoted with $\mathbf{V}^{\mathrm{h}}=\left(\omega^{\mathrm{s}}, \dot{\mathbf{r}}\right)$ as defined in (175). The name indicates that it involves the velocity of the origin of the body-fixed frame but the vectors are resolved in the IFR. The underlying geometric model is the direct product $S O(3) \times \mathbb{R}^{3}$ with the group operation $C_{1} \cdot C_{2}=\left(\mathbf{R}_{1} \mathbf{R}_{2}, \mathbf{r}_{1}+\mathbf{r}_{2}\right)$. The latter is clearly not describing rigid body motions. With $\dot{\mathbf{r}}$ being 'holonomic velocities' the Hamel coefficients are readily deduced from those of the spatial angular velocity (55) as

$$
\gamma_{i j}^{k}= \begin{cases}-\varepsilon_{k i j}, & i, j, k=1,2,3 \\ 0, & i, j, k=4,5,6\end{cases}
$$

\section{Boltzmann-Hamel Equations for the Rigid Body}

\subsection{Kinetic Energy of a Spatially Moving Body}

An arbitrarily located body-fixed reference frame $\mathcal{F}_{\mathrm{b}}$ is used to express the kinetic energy. Denote with $\omega$ the angular velocity of $\mathcal{F}_{\mathrm{b}}$, and with $\mathbf{v}$ the velocity of its origin, both measured in IFR. Let $\mathbf{d}$ be the position vector from the origin of $\mathcal{F}_{b}$ to the COM, and denote with $\Theta^{c}$ the inertia tensor w.r.t. the COM. The latter and all vectors are 
resolved in IFR $\mathcal{F}_{0}$. The kinetic energy is then

$$
\begin{aligned}
T(\boldsymbol{\omega}, \mathbf{v}) & =\frac{1}{2} \boldsymbol{\omega}^{T} \boldsymbol{\Theta} \boldsymbol{\omega}+\frac{m}{2} \mathbf{v}^{T} \mathbf{v}+m \boldsymbol{\omega}^{T} \widetilde{\mathbf{d}} \mathbf{v}=\frac{1}{2}\left(\omega^{T}, \mathbf{v}^{T}\right)\left(\begin{array}{rr}
\boldsymbol{\Theta} & m \tilde{\mathbf{d}} \\
-m \tilde{\mathbf{d}} & m \mathbf{I}
\end{array}\right)\left(\begin{array}{c}
\boldsymbol{\omega} \\
\mathbf{v}
\end{array}\right) \\
& =\frac{1}{2} \omega_{i} \Theta_{i j} \omega_{j}+\frac{m}{2} v_{i} v_{i}+m \varepsilon_{i j k} \omega_{i} d_{j} v_{k}
\end{aligned}
$$

where $\boldsymbol{\Theta}:=\boldsymbol{\Theta}^{c}-m \widetilde{\mathbf{d}} \widetilde{\mathbf{d}}$ is the inertia tensor w.r.t. to the origin of the general body-fixed frame $\mathcal{F}_{\mathrm{b}}$ and $\mathbf{d}$ is the position vector from $\mathcal{F}_{\mathrm{b}}$ to the COM (parallel axis theorem). This is the kinetic energy in terms of the rigid body twist measured at a general point and resolved in the IFR. It is important that the kinetic energy is deduced from the COM motion regardless of which point is used to measure the body twist.

\subsection{Newton-Euler Equations: Body-Fixed Representation of Twists}

\subsubsection{Kinetic Energy}

Denote with $\mathbf{V}^{c}=\left(\omega^{c}, \mathbf{v}^{c}\right)$ the body-fixed twist of a frame $\mathcal{F}_{c}$ at the $\operatorname{COM}\left(\omega^{c}\right.$ is the angular velocity of the body, and $\mathbf{v}^{\mathrm{C}}$ is the velocity of the origin of $\mathcal{F}_{\mathrm{C}}$, both resolved in $\mathcal{F}_{\mathrm{c}}$ ), and denote with $\boldsymbol{\Theta}^{\mathrm{c}}$ the inertia tensor w.r.t. $\mathcal{F}_{\mathrm{c}}$. With the mass $m$, the kinetic energy is $T=\frac{1}{2} \omega^{c T} \Theta^{c} \omega^{c}+\frac{m}{2} \mathbf{v}^{c T} \mathbf{v}^{c}$. Now let $\mathcal{F}_{\mathrm{b}}$ be a general body-fixed frame, with body-fixed twist $\mathbf{V}^{\mathrm{b}}=\left(\boldsymbol{\omega}^{\mathrm{b}}, \mathbf{v}^{\mathrm{b}}\right)$, related to $\mathcal{F}_{\mathrm{c}}$ by the rotation matrix $\mathbf{R}^{\mathrm{bc}}$ and displacement vector ${ }^{\mathrm{b}} \mathbf{d}$ from the origin of $\mathcal{F}_{\mathrm{b}}$ to that of $\mathcal{F}_{\mathrm{c}}$ resolved in $\mathcal{F}_{\mathrm{b}}$. Then $\omega^{\mathrm{c}}=\left(\mathbf{R}^{\mathrm{bc}}\right)^{T} \boldsymbol{\omega}^{\mathrm{b}}$ and $\mathbf{v}^{\mathrm{c}}=\left(\mathbf{R}^{\mathrm{bc}}\right)^{T}\left(\mathbf{v}^{\mathrm{b}}-\widetilde{\boldsymbol{\omega}}^{\mathrm{bb}} \mathbf{d}\right)$. For simplicity, the superscript $\mathrm{b}$ in $\omega^{\mathrm{b}}$ and $\mathbf{v}^{\mathrm{b}}$ will be omitted in the following. The kinetic energy in terms of $\mathbf{V}^{\mathrm{b}}$ is

$$
\begin{aligned}
T(\boldsymbol{\omega}, \mathbf{v}) & =\frac{1}{2}\left(\omega^{T}, \mathbf{v}^{T}\right)\left(\begin{array}{cc}
\boldsymbol{\Theta}^{\mathrm{b}} & m \tilde{\mathbf{d}} \\
-m \tilde{\mathbf{d}} & m \mathbf{I}
\end{array}\right)\left(\begin{array}{c}
\boldsymbol{\omega} \\
\mathbf{v}
\end{array}\right)=\frac{1}{2} \mathbf{V}^{T} \mathbf{M}^{\mathrm{b}} \mathbf{V} \\
& =\frac{1}{2} \boldsymbol{\omega}^{T} \boldsymbol{\Theta}^{\mathrm{b}} \boldsymbol{\omega}+\frac{m}{2} \mathbf{v}^{T} \mathbf{v}+m \boldsymbol{\omega}^{T} \tilde{\mathbf{d}} \\
& =\frac{1}{2} \omega_{i} \Theta_{i j}^{\mathrm{b}} \omega_{j}+\frac{m}{2} v_{i} v_{i}+m \varepsilon_{i j k} \omega_{i} d_{j} v_{k}
\end{aligned}
$$

where $\mathbf{M}^{\mathrm{b}}$ is the constant mass matrix expressed in body-fixed frame with $\boldsymbol{\Theta}^{\mathrm{b}}=$ $\mathbf{R}^{\mathrm{bc}} \boldsymbol{\Theta}^{\mathrm{c}}\left(\mathbf{R}^{\mathrm{bc}}\right)^{T}-m \tilde{\mathbf{d}} \widetilde{\mathbf{d}}$. The kinetic energy (85) is invariant under change of bodyfixed reference frame, i.e. left-invariant under action of $S E(3)$.

\subsubsection{Boltzmann-Hamel equations}

For the body-fixed representation of twists, the kinetic energy (85) does not depend on the pose of the body. Using the Hamel coefficients (72) yields to the BH-equations (14) (omitting superscript b)

$$
\frac{d}{d t} \frac{\partial T}{\partial \omega_{i}}+\frac{\partial T}{\partial \omega_{k}} \varepsilon_{k i j} \omega_{j}+\frac{\partial T}{\partial v_{k}} \varepsilon_{k i j} v_{j}=\tau_{i}, \quad a=i=1,2,3
$$




$$
\frac{d}{d t} \frac{\partial T}{\partial v_{i}}+\frac{\partial T}{\partial v_{k}} \varepsilon_{k i j} \omega_{j}=f_{i}, \quad a=i+3=4,5,6
$$

where $\tau_{i}$ and $f_{i}$ are the components of the applied torque and force expressed in the reference frame $\mathcal{F}_{\mathrm{b}}$. Inserting the derivatives

$$
\begin{aligned}
\frac{\partial T}{\partial \omega_{i}} & =\Theta_{i j}^{\mathrm{b}} \omega_{j}+m \varepsilon_{i j k} d_{j} v_{k}, \quad \frac{d}{d t} \frac{\partial T}{\partial \omega_{i}}=\Theta_{i j}^{\mathrm{b}} \dot{\omega}_{j}+m \varepsilon_{i j k} d_{j} \dot{v}_{k} \\
\frac{\partial T}{\partial v_{i}} & =m\left(v_{i}+\varepsilon_{i j k} \omega_{j} d_{k}\right), \quad \frac{d}{d t} \frac{\partial T}{\partial v_{i}}=m\left(\dot{v}_{i}+\varepsilon_{i j k} \dot{\omega}_{j} d_{k}\right)
\end{aligned}
$$

in (86) yields

$$
\Theta_{i j} \dot{\omega}_{j}+m \varepsilon_{i j k} d_{j} \dot{v}_{k}+\varepsilon_{i j k} \omega_{j} \Theta_{k l} \omega_{l}+m \varepsilon_{i j k}\left(\omega_{j} \varepsilon_{k r s} d_{r} v_{s}+v_{j} \varepsilon_{k r s} \omega_{r} d_{s}\right)=\tau_{i}
$$

The last term can be simplified, invoking the Jacobi identity $\varepsilon_{i j k} \varepsilon_{k r s}+\varepsilon_{i s k} \varepsilon_{k j r}=$ $-\varepsilon_{i r k} \varepsilon_{k s j}$. With this, and introducing (88) in the second Eq. (87) yields

$$
\begin{aligned}
\Theta_{i j}^{\mathrm{b}} \dot{\omega}_{j}+\varepsilon_{i j k} \omega_{j} \Theta_{k l}^{\mathrm{b}} \omega_{l}+m \varepsilon_{i j k} d_{j}\left(\dot{v}_{k}+\varepsilon_{k r s} \omega_{s} v_{r}\right) & =\tau_{i} \\
m\left(\dot{v}_{i}+\varepsilon_{i j k} \omega_{j} v_{k}+\left(\varepsilon_{i j s} \dot{\omega}_{j}+\varepsilon_{i j k} \varepsilon_{k r s} \omega_{j} \omega_{r}\right) d_{s}\right) & =f_{i} .
\end{aligned}
$$

These are the NE-equations w.r.t. to an arbitrary body-fixed frame, which read in vector form

$$
\begin{aligned}
\boldsymbol{\Theta}^{\mathrm{b}} \dot{\boldsymbol{\omega}}^{\mathrm{b}}+\widetilde{\boldsymbol{\omega}}^{\mathrm{b}} \boldsymbol{\Theta}^{\mathrm{b}} \boldsymbol{\omega}^{\mathrm{b}}+m \widetilde{\mathbf{d}}\left(\dot{\mathbf{v}}^{\mathrm{b}}+\widetilde{\boldsymbol{\omega}}^{\mathrm{b}} \mathbf{v}^{\mathrm{b}}\right) & =\boldsymbol{\tau}^{\mathrm{b}} \\
m\left(\dot{\mathbf{v}}^{\mathrm{b}}+\widetilde{\boldsymbol{\omega}}^{\mathrm{b}} \mathbf{v}^{\mathrm{b}}+\left(\dot{\widetilde{\boldsymbol{\omega}}}^{\mathrm{b}}+\widetilde{\boldsymbol{\omega}}^{\mathrm{b}} \widetilde{\boldsymbol{\omega}}^{\mathrm{b}}\right) \mathbf{d}\right) & =\mathbf{f}^{\mathrm{b}} .
\end{aligned}
$$

They simplify if the body-fixed reference frame is located at the COM, i.e. if $\mathbf{d}=\mathbf{0}$.

\subsubsection{BH-Equations as Euler-Poincaré Equations on SE (3)}

Equation (91) can be written in the compact form (Müller 2018)

$$
\mathbf{M}^{\mathrm{b}} \dot{\mathbf{V}}^{\mathrm{b}}-\mathbf{a d}_{\mathbf{V}^{\mathrm{b}}}^{T} \mathbf{M}^{\mathrm{b}} \mathbf{V}^{\mathrm{b}}=\mathbf{W}^{\mathrm{b}}
$$

where $\mathbf{W}^{\mathrm{b}}=\left(\boldsymbol{\tau}^{\mathrm{b}}, \mathbf{f}^{\mathrm{b}}\right) \in s e^{*}(3)$ is the body-fixed representation of the wrench acting on the body. These are the Euler-Poincaré equations (41) for the left-invariant kinetic energy (85) (invariant w.r.t. choice of IFR). They can be written as

$$
\dot{\Pi}^{\mathrm{b}}-\mathbf{a d}_{\mathbf{v}^{\mathrm{b}}}^{T} \boldsymbol{\Pi}^{\mathrm{b}}=\mathbf{W}^{\mathrm{b}}
$$

in terms of the body-fixed momentum $\Pi^{\mathrm{b}}:=\mathbf{M}^{\mathrm{b}} \mathbf{V}^{\mathrm{b}} \in s e^{*}(3)$, with which the kinetic energy is $T\left(\mathbf{V}^{\mathrm{b}}\right):=\frac{1}{2} \Pi^{\mathrm{b}} \mathbf{V}^{\mathrm{b}}$. 


\subsection{Newton-Euler Equations: Spatial Representation of Twists}

\subsubsection{Kinetic Energy}

The peculiarity of the spatial twist (160) is that $\mathbf{v}^{s}=\dot{\mathbf{r}}-\widetilde{\mathbf{r}}^{\mathrm{s}} \boldsymbol{\omega}^{\mathrm{s}}$ is the velocity of the point of the body which is momentarily coinciding with the origin of the IFR (recall that $\mathbf{r}$ is the position vector of the body-fixed frame pressed in IFR). Denote with $\mathbf{d}$ the vector from the body-fixed frame to the COM. The velocity of the COM, expressed in IFR, is then $\mathbf{v}^{s}+(\widetilde{\mathbf{d}}+\widetilde{\mathbf{r}}) \widetilde{\boldsymbol{\omega}}^{\mathrm{s}}$, and the expression for the kinetic energy in terms of spatial twists defined in (160) is

$$
\begin{aligned}
T\left(\boldsymbol{\varphi}, \mathbf{r}, \omega^{\mathrm{s}}, \mathbf{v}^{\mathrm{s}}\right) & =\frac{1}{2}\left(\omega^{\mathrm{s} T}, \mathbf{v}^{\mathrm{s} T}\right)\left(\begin{array}{cc}
\boldsymbol{\Theta}^{\mathrm{s}} & m(\widetilde{\mathbf{d}}+\widetilde{\mathbf{r}}) \\
-m(\widetilde{\mathbf{d}}+\widetilde{\mathbf{r}}) & m \mathbf{I}
\end{array}\right)\left(\begin{array}{c}
\omega^{\mathrm{s}} \\
\mathbf{v}^{\mathrm{s}}
\end{array}\right)=\frac{1}{2} \mathbf{V}^{\mathrm{s} T} \mathbf{M}^{\mathrm{s}} \mathbf{V}^{\mathrm{s}} \\
& =\frac{1}{2} \omega_{i}^{\mathrm{s}} \Theta_{i j}^{\mathrm{s}} \omega_{j}^{\mathrm{s}}+\frac{m}{2} v_{i}^{\mathrm{s}} v_{i}^{\mathrm{s}}+m \varepsilon_{i j k} \omega_{i}^{\mathrm{s}}\left(d_{j}+r_{j}\right) v_{k}^{\mathrm{s}}
\end{aligned}
$$

where $\boldsymbol{\Theta}^{\mathrm{s}}={ }^{\mathrm{s}} \boldsymbol{\Theta}^{\mathrm{c}}-m(\tilde{\mathbf{d}}+\widetilde{\mathbf{r}})$ is the inertia tensor w.r.t. the body-fixed point that coincides with the IFR origin resolved in IFR. Therein, ${ }^{5} \boldsymbol{\Theta}^{c}=\mathbf{R} \Theta^{C} \mathbf{R}^{T}$ is the inertia tensor w.r.t. the COM, resolved in the IFR, where $\mathbf{R}$ is the rotation matrix in (157) transforming from body-fixed frame $\mathcal{F}_{\mathrm{b}}$ to IFR $\mathcal{F}_{0}$. Its components are

$$
\Theta_{i j}^{\mathrm{s}}(\boldsymbol{\varphi})={ }^{\mathrm{s}} \Theta_{i j}^{\mathrm{c}}(\boldsymbol{\varphi})-m \varepsilon_{i k l} \varepsilon_{l r j}\left(d_{k}+r_{k}\right)\left(d_{r}+r_{r}\right) .
$$

Instead of writing $C \in S E(3)$, local coordinates $\left(q^{a}\right)=\left(\varphi^{i}, r_{i}\right)$ are used in (94) to express the rigid body pose. The superscript $s$ in $\omega^{S}$ and $\mathbf{v}^{S}$ will be omitted in the following. All vectors are resolved in the IFR. The kinetic energy (94) is invariant under change of IFR, i.e. right-invariant under action of $S E(3)$.

\subsubsection{Boltzmann-Hamel Equations}

With the Hamel coefficients (82) and the kinetic energy, the BH-equations (14) are (omitting the superscript $\mathrm{s}$ of $v_{i}^{\mathrm{s}}$ and $\omega_{i}^{\mathrm{s}}$ )

$$
\begin{aligned}
& \frac{d}{d t} \frac{\partial T}{\partial \omega_{i}}-\frac{\partial T}{\partial \varphi^{j}} \frac{\partial \dot{\varphi}^{j}}{\partial \omega_{i}} \\
& -\frac{\partial T}{\partial r_{j}} \frac{\partial \dot{r}_{j}}{\partial \omega_{i}}-\frac{\partial T}{\partial \omega_{k}} \varepsilon_{k i j} \omega_{j}-\frac{\partial T}{\partial v_{k}} \varepsilon_{k i j} v_{j}=\tau_{i}, \quad a=i=1,2,3 \\
& \frac{d}{d t} \frac{\partial T}{\partial v_{i}}-\frac{\partial T}{\partial r_{i}}-\frac{\partial T}{\partial v_{k}} \varepsilon_{k i j} \omega_{j}=f_{i}, \quad a=i+3=4,5,6 .
\end{aligned}
$$

For the subsequent derivation, the following relations will be necessary, which follow from the definition of the spatial twist (160) and from (149):

$$
\dot{r}_{i}=v_{i}-\varepsilon_{i j k} r_{j} \omega_{k}, \quad \frac{\partial \dot{r}_{j}}{\partial \omega_{i}}=-\varepsilon_{j k i} r_{k}, \quad \frac{\partial \dot{\varphi}_{j}}{\partial \omega_{i}}=\Psi_{i}^{j} .
$$


Therewith the first set of Eq. (96), describing the angular dynamics, is

$$
\frac{d}{d t} \frac{\partial T}{\partial \omega_{i}}-\frac{\partial T}{\partial \varphi^{j}} \Psi_{i}^{j}+\frac{\partial T}{\partial r_{j}} \varepsilon_{j k i} r_{k}-\frac{\partial T}{\partial \omega_{k}} \varepsilon_{k i j} \omega_{j}-\frac{\partial T}{\partial v_{k}} \varepsilon_{k i j} v_{j}=\tau_{i}
$$

From the kinetic energy (94) follows immediately

$$
\frac{\partial T}{\partial \omega_{i}}=\Theta_{i j}^{s} \omega_{j}+m \varepsilon_{i j k}\left(d_{j}+r_{j}\right) v_{k}
$$

and inserting (95) yields

$$
\begin{aligned}
\frac{d}{d t} \frac{\partial T}{\partial \omega_{i}} & =\Theta_{i j}^{s} \dot{\omega}_{j}+\left({ }^{s} \dot{\Theta}_{i j}^{c}-m \varepsilon_{i k l} \varepsilon_{l r j}\left(\left(d_{k}+r_{k}\right)\left(\dot{d}_{r}+\dot{r}_{r}\right)+\left(\dot{d}_{k}+\dot{r}_{k}\right)\left(d_{r}+r_{r}\right)\right)\right) \omega_{j} \\
& +m \varepsilon_{i j k}\left(\left(d_{j}+r_{j}\right) \dot{v}_{k}+\left(\dot{d}_{j}+\dot{r}_{j}\right) v_{k}\right) .
\end{aligned}
$$

Introducing the relations $\dot{d}_{j}=\varepsilon_{j l r} \omega_{l} d_{r}$ and ${ }^{\mathrm{s}} \dot{\Theta}_{i j}^{\mathrm{c}}=\left(\varepsilon_{i k l}{ }^{\mathrm{s}} \Theta_{l j}^{\mathrm{c}}-{ }^{\mathrm{s}} \Theta_{i l}^{\mathrm{c}} \varepsilon_{k l j}\right) \omega_{k}$ along with (98) leads to

$$
\begin{aligned}
\frac{d}{d t} \frac{\partial T}{\partial \omega_{i}}= & \Theta_{i j}^{\mathrm{s}} \dot{\omega}_{j}+\varepsilon_{i k l} \omega_{k}^{\mathrm{s}} \Theta_{l j}^{\mathrm{c}} \omega_{j}+m \varepsilon_{i j k}\left(d_{j}+r_{j}\right) \dot{v}_{k}-m \varepsilon_{i k j} \varepsilon_{j l r}\left(d_{k}+r_{k}\right) v_{l} \omega_{r} \\
& +m \varepsilon_{i k l}\left(\varepsilon_{l j r} \varepsilon_{r s p}\left(d_{k}+r_{k}\right) \omega_{j} \omega_{s}\left(d_{p}+r_{p}\right)\right. \\
& \left.-\varepsilon_{k s p} \varepsilon_{l r j} \omega_{s}\left(d_{p}+r_{p}\right)\left(d_{r}+r_{r}\right) \omega_{j}\right)
\end{aligned}
$$

The term in the second line of (101) can be summarized as $-m \varepsilon_{i k l} \varepsilon_{l s p} \varepsilon_{p r j} \omega_{k}\left(d_{s}+\right.$ $\left.r_{s}\right) \omega_{j}$, and (101) simplifies to

$$
\frac{d}{d t} \frac{\partial T}{\partial \omega_{i}}=\Theta_{i j}^{\mathrm{s}} \dot{\omega}_{j}+\varepsilon_{i k l} \omega_{k} \Theta_{l j}^{\mathrm{s}} \omega_{j}+m \varepsilon_{i j k}\left(d_{j}+r_{j}\right)\left(\dot{v}_{k}+\varepsilon_{j l r} \omega_{l} v_{r}\right) .
$$

The partial derivative of the kinetic energy (94) w.r.t. $\varphi$ follows with (95) as

$$
\begin{aligned}
\frac{\partial T}{\partial \varphi^{i}}= & \frac{1}{2} \omega_{k} \frac{\partial^{s} \Theta_{k j}^{c}}{\partial \varphi^{i}} \omega_{j}+\frac{1}{2} m \varepsilon_{l j k} \frac{\partial d_{j}}{\partial \varphi^{i}} v_{k} \omega_{l} \\
= & \frac{1}{2} \omega_{k}\left(\frac{\partial^{s} \Theta_{k j}^{c}}{\partial \varphi^{i}}-m \varepsilon_{k s l} \varepsilon_{l r j}\left(\frac{\partial d_{s}}{\partial \varphi^{i}}\left(d_{r}+r_{r}\right)+\left(d_{s}+r_{s}\right) \frac{\partial d_{r}}{\partial \varphi^{i}}\right)\right) \omega_{j} \\
& +m \omega_{k} \varepsilon_{k j l} \frac{\partial d_{j}}{\partial \varphi^{i}} v_{l} .
\end{aligned}
$$

Introducing $\frac{\partial d_{j}}{\partial \varphi^{i}}=\varepsilon_{j r s} \Omega_{r i} d_{s}$ and $\frac{\partial^{\mathrm{s}} \Theta_{k j}^{c}}{\partial \varphi^{i}}=\varepsilon_{k l s} \Omega_{l i}{ }^{\mathrm{s}} \Theta_{s j}^{\mathrm{c}}-{ }^{\mathrm{s}} \Theta_{k s}^{\mathrm{c}} \varepsilon_{s l j} \Omega_{l i}$, some straightforward manipulation yields

$\frac{\partial T}{\partial \varphi^{i}}=-\Omega_{j i} \varepsilon_{j s l} \omega_{s}{ }^{\mathrm{s}} \Theta_{l k}^{\mathrm{c}} \omega_{k}+m \omega_{k} \varepsilon_{k j l} \varepsilon_{j r s} \Omega_{r i} d_{s} v_{l}-m \omega_{k} \varepsilon_{k s l} \varepsilon_{s p n} \Omega_{p i} d_{n} \varepsilon_{l r j}\left(d_{r}+r_{r}\right) \omega_{j}$. 
The derivative w.r.t. $r_{k}$ can be simplified with $\varepsilon_{i k l} \varepsilon_{l r j}+\varepsilon_{i r l} \varepsilon_{l k j}=2 \varepsilon_{i k l} \varepsilon_{l r j}+\varepsilon_{l r j} \varepsilon_{l k r}$ to yield

$$
\begin{aligned}
\frac{\partial T}{\partial r_{k}} & =m \varepsilon_{i k l} \omega_{i} v_{j}-\frac{m}{2} \omega_{i}\left(\varepsilon_{i k l} \varepsilon_{l r j}\left(d_{r}+r_{r}\right)+\varepsilon_{i r l} \varepsilon_{l k j}\left(d_{r}+r_{r}\right)\right) \omega_{j} \\
& =m \varepsilon_{i k l} \omega_{i} v_{j}-m \omega_{i} \varepsilon_{i k l} \varepsilon_{l r j}\left(d_{r}+r_{r}\right) \omega_{j} .
\end{aligned}
$$

Finally, it is readily found that

$$
\frac{\partial T}{\partial v_{k}}=m\left(\dot{v}_{i}+\varepsilon_{i j k} \dot{\omega}_{j}\left(d_{k}+r_{k}\right)+\varepsilon_{i j k} \omega_{j}\left(v_{k}+\varepsilon_{k l r} \omega_{l}\left(d_{r}+r_{r}\right)\right)\right) .
$$

Inserting (100), (104), (105), and (106) into the BH-equation (96), noting that $\Omega_{i k} \Psi_{j}^{k}=$ $\delta_{i j}$, and summarizing terms, yields

$$
\begin{aligned}
\tau_{i} & =\frac{d}{d t} \frac{\partial T}{\partial \omega_{i}} \\
& (*)+\omega_{s}\left(\varepsilon_{i s l}^{\mathrm{s}} \Theta_{l k}^{\mathrm{c}} \omega_{k}-\varepsilon_{i s k}^{\mathrm{s}} \Theta_{k l}^{\mathrm{c}} \omega_{l}\right)-m v_{k} \varepsilon_{k i j} v_{j} \\
& (* *)+m \omega_{k} \varepsilon_{k s l} \varepsilon_{s i n} d_{n} \varepsilon_{l r j}\left(d_{r}+r_{r}\right) \omega_{j}+m \omega_{k} \varepsilon_{s j l} \varepsilon_{j i p} r_{p} \varepsilon_{l r k}\left(d_{r}+r_{r}\right) \omega_{k} \\
& \quad+m \omega_{j} \varepsilon_{i j k} \varepsilon_{k s l}\left(d_{s}+r_{s}\right) \varepsilon_{l r p}\left(d_{r}+r_{r}\right) \omega_{p} \\
& (* * *)-m \omega_{j} \varepsilon_{i j k} \varepsilon_{k r s}\left(d_{r}+r_{r}\right) v_{s}-m v_{j} \varepsilon_{i j k} \varepsilon_{k r s} \omega_{r}\left(d_{s}+r_{s}\right) \\
& (* * * *)+m d_{s} \varepsilon_{i s j} \varepsilon_{j k l} \omega_{k} v_{l}+m r_{k} \varepsilon_{i k j} \varepsilon_{j r s} \omega_{r} v_{s} .
\end{aligned}
$$

The expression (102) was not inserted on purpose. The terms $(*)$ and $(* *)$ clearly vanish. Due to the Jacobi identity, $(* * *)$ is equal to $m \varepsilon_{i j k}\left(d_{j}+r_{j}\right) \varepsilon_{k r s} v_{r} \omega_{s}$. Summarizing the term $(* * * *)$ as $m \varepsilon_{i j k}\left(d_{j}+r_{j}\right) \varepsilon_{k r s} \omega_{r} v_{s}$ shows that $(* * *)+(* * * *)=0$. Equation (96) thus attains the simple form

$$
\begin{aligned}
\tau_{i} & =\frac{d}{d t} \frac{\partial T}{\partial \omega_{i}} \\
& =\Theta_{i j}^{\mathrm{s}} \dot{\omega}_{j}+\varepsilon_{i k l} \omega_{k} \Theta_{l j}^{\mathrm{s}} \omega_{j}+m \varepsilon_{i j k}\left(d_{j}+r_{j}\right)\left(\dot{v}_{k}+\varepsilon_{j l r} \omega_{l} v_{r}\right), \quad i=1,2,3 .
\end{aligned}
$$

The second set of Eq. (97) yields, with (105) and (106), and after simplification

$$
f_{i}=m\left(\dot{v}_{i}-\varepsilon_{i j k}\left(d_{j}+r_{j}\right) \dot{\omega}_{k}+\varepsilon_{i j k} \omega_{j}\left(v_{k}+\varepsilon_{k l r} \omega_{l}\left(d_{r}+r_{r}\right)\right)\right), \quad i=1,2,3 .
$$

Equations. (108) and (109) are the Newton-Euler equations governing the rigid body dynamics in terms of the spatial twist. They are in vector form

$$
\begin{aligned}
\boldsymbol{\Theta}^{\mathrm{s}} \dot{\boldsymbol{\omega}}^{\mathrm{s}}+\widetilde{\boldsymbol{\omega}}^{\mathrm{s}} \boldsymbol{\Theta}^{\mathrm{s}} \boldsymbol{\omega}^{\mathrm{s}}+m(\widetilde{\mathbf{d}}+\widetilde{\mathbf{r}})\left(\dot{\mathbf{v}}^{\mathrm{s}}+\widetilde{\boldsymbol{\omega}}^{\mathrm{s}} \mathbf{v}^{\mathrm{s}}\right) & =\boldsymbol{\tau}^{\mathrm{s}} \\
m\left(\dot{\mathbf{v}}^{\mathrm{s}}+\widetilde{\boldsymbol{\omega}}^{\mathrm{s}} \mathbf{v}^{\mathrm{s}}+\left(\dot{\widetilde{\boldsymbol{\omega}}}^{\mathrm{s}}+\widetilde{\boldsymbol{\omega}}^{\mathrm{s}} \widetilde{\boldsymbol{\omega}}^{\mathrm{s}}\right)(\mathbf{d}+\mathbf{r})\right) & =\mathbf{f}^{\mathrm{s}}
\end{aligned}
$$

These equations cannot be simplified, as (91) can be by setting $\mathbf{d}=\mathbf{0}$, since the COM cannot be used as reference point. 


\subsubsection{BH-Equations as Euler-Lagrange Equations on SE (3)}

Also the spatial NE equations $(108,109)$ can be written in compact vector form

$$
\mathbf{M}^{\mathrm{s}} \dot{\mathbf{V}}^{\mathrm{s}}-\mathbf{a d}_{\mathbf{V}^{\mathrm{s}}}^{T} \mathbf{M}^{\mathrm{s}} \mathbf{V}^{\mathrm{s}}=\mathbf{W}^{\mathrm{s}}
$$

where $\mathbf{W}^{s}=\left(\boldsymbol{\tau}^{s}, \mathbf{f}^{s}\right) \in s e^{*}(3)$ is the applied wrench in spatial representation. These are the Euler-Lagrange equations (39) for the right-invariant kinetic energy. Although (112) seem formally identical to (41), and to (92) in particular, they are not the EulerPoincaré equations (which would require a positive sign in (112). The kinetic energy in terms of spatial twists is right invariant (independent from the choice of bodyfixed frame), but it depends on the orientation and thus on the pose of the body: $T=T\left(C, \mathbf{V}^{\mathrm{s}}\right)$.

Remark 8 Equations. (110) and (111) can be readily derived from the conservation of the spatial momentum $\Pi^{\mathrm{s}}=\mathbf{M}^{\mathrm{s}} \mathbf{V}^{\mathrm{s}}$, using $\dot{\mathbf{M}}^{\mathrm{s}}=-\mathbf{M}^{\mathrm{s}} \mathbf{a d}_{\mathbf{V}^{\mathrm{s}}}-\operatorname{ad}_{\mathbf{V}^{\mathrm{s}}}^{T} \mathbf{M}^{\mathrm{s}}$, as

$$
\dot{\Pi}^{\mathrm{s}}=\mathbf{W}^{\mathrm{s}}
$$

Beside its simplicity, the spatial form (113) is also computationally advantageous. An important advantage is that momentum conserving numerical integration schemes can be easily derived, including Lie group integration schemes. This was discussed in (Borri et al. 2001, 2003; Bottasso and Borri 1998). In this context, the formulation in terms of spatial twists was called fixed-pole formulation.

\subsection{Newton-Euler Equations: Hybrid Representation of Twists}

\subsubsection{Kinetic Energy}

The kinetic energy in terms of the hybrid twist (175) is

$$
\begin{aligned}
T\left(\boldsymbol{\varphi}, \omega^{\mathrm{s}}, \dot{\mathbf{r}}\right) & =\frac{1}{2}\left(\omega^{\mathrm{s} T}, \dot{\mathbf{r}}^{T}\right)\left(\begin{array}{cc}
{ }^{\mathrm{s}} \boldsymbol{\Theta}^{\mathrm{b}} & m \widetilde{\mathbf{d}} \\
-m \widetilde{\mathbf{d}} & m \mathbf{I}
\end{array}\right)\left(\begin{array}{c}
\omega^{\mathrm{s}} \\
\dot{\mathbf{r}}
\end{array}\right)=\frac{1}{2} \mathbf{V}^{\mathrm{h} T} \mathbf{M}^{\mathrm{h}} \mathbf{V}^{\mathrm{h}} \\
& =\frac{1}{2} \omega^{\mathrm{s} T \mathrm{~s}} \boldsymbol{\Theta}^{\mathrm{b}} \omega^{\mathrm{s}}+\frac{m}{2} \dot{\mathbf{r}}^{T} \dot{\mathbf{r}}+m \omega^{\mathrm{s} T} \tilde{\mathbf{d}} \dot{\mathbf{r}} \\
& =\frac{1}{2} \omega_{i}^{\mathrm{s}} \Theta_{i j}^{\mathrm{b}} \omega_{j}^{\mathrm{s}}+\frac{m}{2} \dot{r}_{i} \dot{r}_{i}+m \varepsilon_{i j k} \omega_{i}^{\mathrm{s}} d_{j} \dot{r}_{k}
\end{aligned}
$$

where ${ }^{\mathrm{s}} \boldsymbol{\Theta}^{\mathrm{b}}=\mathbf{R} \boldsymbol{\Theta}^{\mathrm{b}} \mathbf{R}^{T}$ is the inertia tensor w.r.t. the (arbitrarily located) body-fixed frame (superscript $b$ ) resolved in the IFR (superscript $s$ ). All vectors are resolved in the IFR. The superscripts of $\omega^{\mathrm{s}}$ and ${ }^{\mathrm{s}} \boldsymbol{\Theta}^{\mathrm{b}}$ will be omitted. The kinetic energy (114) is right-invariant under action of $S O(3) \times \mathbb{R}^{3}$, i.e. it is invariant w.r.t. rotations of IFR and displacements of its origin taking place independently. 


\subsubsection{Boltzmann-Hamel Equations}

With the Hamel coefficients (83) being zero for $a, b, c=4,5$, 6, and with kinetic energy being independent from $\mathbf{r}$, the $\mathrm{BH}$-equations are

$$
\begin{aligned}
\frac{d}{d t} \frac{\partial T}{\partial \omega_{i}}-\frac{\partial T}{\partial \varphi^{j}} \Psi_{i}^{j}+\frac{\partial T}{\partial \omega_{k}} \gamma_{i j}^{k} \omega_{j} & =\tau_{i}^{\mathrm{s}}, \quad & a & =i=1,2,3 \\
\frac{d}{d t} \frac{\partial T}{\partial \dot{r}_{i}} & =f_{i}^{\mathrm{h}}, & & a=i+3=4,5,6
\end{aligned}
$$

where $f_{i}^{\mathrm{h}}=R_{i j} f_{j}^{\mathrm{b}}$ are the coordinates of the force applied at the origin of the bodyfixed reference frame resolved in the IFR, and $\tau_{i}^{\mathrm{s}}$ coordinates of the spatial torque as in (108). From (114) follows that $\frac{\partial T}{\partial \omega_{i}}=\Theta_{i j} \omega_{j}+m \varepsilon_{i j k} d_{j} \dot{r}_{k}$ and thus

$$
\begin{aligned}
\frac{d}{d t} \frac{\partial T}{\partial \omega_{i}} & =\frac{d}{d t}\left(\Theta_{i j} \omega_{j}\right)+m \varepsilon_{i j k}\left(d_{j} \ddot{r}_{k}+\dot{d}_{j} \dot{r}_{k}\right) \\
& =\frac{d}{d t}\left(\Theta_{i j} \omega_{j}\right)+m \varepsilon_{i j k} d_{j} \ddot{r}_{k}+m \varepsilon_{i j k} \varepsilon_{j r l} \omega_{r} d_{l} \dot{r}_{k}
\end{aligned}
$$

where $\dot{d}_{j}=\varepsilon_{j r l} \omega_{r} d_{l}$ has been used. Using $\frac{\partial d_{j}}{\partial \varphi_{i}}=\varepsilon_{j r s} \Omega_{r i} d_{s} \dot{r}_{k}$ yields the partial derivatives

$$
\begin{aligned}
\frac{\partial T}{\partial \varphi_{i}} & =\frac{1}{2} \omega_{k} \frac{\partial \Theta_{k j}}{\partial \varphi^{i}} \omega_{j}+m \varepsilon_{l j k} \varepsilon_{j r s} \omega_{l} \Omega_{r i} d_{s} \dot{r}_{k} \\
\frac{d}{d t} \frac{\partial T}{\partial \dot{r}_{i}} & =m \ddot{r}_{i}+m \varepsilon_{i j k}\left(\dot{\omega}_{j} d_{k}+\omega_{j} \dot{d}_{k}\right) \\
& =m\left(\ddot{r}_{i}+\varepsilon_{i j k} \dot{\omega}_{j} d_{k}+\varepsilon_{i j k} \varepsilon_{k r s} \omega_{j} \omega_{r} d_{s}\right) .
\end{aligned}
$$

The BH-equations (115) for $i=1,2,3$ are thus, with $\gamma_{i j}^{k}=-\varepsilon_{k i j}$ in (83),

$$
\begin{aligned}
\tau_{i}= & \frac{d}{d t}\left(\Theta_{i j} \omega_{j}\right)-\frac{1}{2} \omega_{k} \frac{\partial \Theta_{k r}}{\partial \varphi^{j}} \omega_{r} \Psi_{i}^{j}-\varepsilon_{k i j} \Theta_{k s} \omega_{s} \omega_{j} \\
& +m \varepsilon_{i j k} d_{j} \ddot{r}_{k} \\
& +m \varepsilon_{i j k} \varepsilon_{j r l} \omega_{r} d_{l} \dot{r}_{k}-m \varepsilon_{l p k} \varepsilon_{p r s} \omega_{l} \Omega_{r j} d_{s} \dot{r}_{k} \Psi_{i}^{j}-m \varepsilon_{k i j} \varepsilon_{k r s} d_{r} \dot{r}_{s} \omega_{j}
\end{aligned}
$$

With $\Omega_{r j} \Psi_{i}^{j}=\delta_{r i}$, the last line in (121) can be rearranged as $-m\left(\varepsilon_{i j k} \varepsilon_{k r s}+\varepsilon_{i s k} \varepsilon_{k j l}+\right.$ $\left.\varepsilon_{i r k} \varepsilon_{k s j}\right) \dot{r}_{j} \omega_{r} d_{s} \equiv 0$, and thus vanishes identically. The right hand side of the first line in (121) is identical to the left hand side of (65), which yields the Euler-equations (66). Thus, the first set of BH-equations ( 115) are

$$
{ }^{\mathrm{s}} \Theta_{i j}^{\mathrm{b}} \dot{\omega}_{j}^{\mathrm{s}}+\varepsilon_{i k l} \omega_{k}^{\mathrm{ss}} \Theta_{l j}^{\mathrm{b}} \omega_{j}^{\mathrm{s}}+m \varepsilon_{i j k} d_{j} \ddot{r}_{k}=\tau_{i}^{\mathrm{s}} .
$$


The second set of BH-equations (65) follows immediately from (120) as

$$
m\left(\ddot{r}_{i}+\varepsilon_{i j k} \dot{\omega}_{j}^{\mathrm{s}} d_{k}+\varepsilon_{i j k} \varepsilon_{k r s} \omega_{j}^{\mathrm{s}} \omega_{r}^{\mathrm{s}} d_{s}\right)=f_{i}^{\mathrm{h}} .
$$

These are the Newton-Euler equations in terms of the hybrid twists, i.e. spatial angular and linear velocity of an arbitrarily located body-fixed reference frame. In vector form they are

$$
\begin{aligned}
{ }^{\mathrm{s}} \boldsymbol{\Theta}^{\mathrm{b}} \dot{\boldsymbol{\omega}}^{\mathrm{s}}+\widetilde{\boldsymbol{\omega}}^{\mathrm{ss}} \boldsymbol{\Theta}^{\mathrm{b}} \boldsymbol{\omega}^{\mathrm{s}}+m \widetilde{\mathbf{d}} \ddot{\mathbf{r}}=\boldsymbol{\tau}^{\mathrm{s}} \\
m\left(\ddot{\mathbf{r}}+\left(\dot{\tilde{\boldsymbol{\omega}}}^{\mathrm{s}}+\widetilde{\boldsymbol{\omega}}^{\mathrm{s}} \widetilde{\boldsymbol{\omega}}^{\mathrm{s}}\right) \mathbf{d}\right)=\mathbf{f}^{\mathrm{h}} .
\end{aligned}
$$

These are equivalent to the explicit form (91) of the body-fixed Newton-Euler equations when expressed in IFR, noting that the acceleration of the origin of the body-fixed frame in body-fixed representation is $\dot{\mathbf{v}}^{\mathrm{b}}+\widetilde{\boldsymbol{\omega}}^{\mathrm{b}} \mathbf{v}^{\mathrm{b}}$.

Remark 9 Equation (124) are the Euler-Lagrange equations for the right-invariant Lagrangian on $S O(3) \times \mathbb{R}^{3}$. Using the direct product as rigid body configuration space (in which rotations and translations are assumed decoupled) has consequences when Lie group integration schemes are applied to the rigid body equations (Müller 2016; Müller and Terze 2014), since $S O(3) \times \mathbb{R}^{3}$ does not describe frame transformations, i.e. rigid body motions.

\subsection{A Nonholonomically Constrained Rigid Body: Ball Rolling on a Horizontal Plane}

As an example for a constrained rigid body, a solid ball rolling without slipping on a horizontal plane is considered. It is assumed that the COM is at the geometric center of the ball. Yet the mass distribution is assumed inhomogenous so that the ball possess different principle moments of inertia. This is a simplification of the classical problem of an inhomogenous rolling ball treated in detail already by Chaplygin (1897a, b, 1903) and Woronetz (1910).

\subsubsection{Rolling-Without-Slipping Constraint}

A solid ball with radius $\rho$ rolling on a plane without slipping (Fig. 1) is a nonholonomically constrained system. The body-fixed frame $\mathcal{F}_{\mathrm{b}}$ is attached at the COM of the ball. Denote with $\mathbf{r}=\left(r_{1}, r_{2}, r_{3}\right)$ the position vector of its COM represented in the IFR $\mathcal{F}_{0}$, and with $\varphi=\left(\varphi^{1}, \varphi^{2}, \varphi^{3}\right)$ the vector of three parameters describing the orientation. The $n=6$ coordinates describing the pose of the ball are thus $\mathbf{q}=\left(q^{1}, \ldots, q^{6}\right)=(\varphi, \mathbf{r})$. The hybrid twist $\mathbf{V}=\left(\omega^{\mathrm{s}}, \dot{\mathbf{r}}\right)$ is used to describe the velocity of the ball, where the spatial angular velocity is determined by (153). The superscript $\mathbf{s}$ is omitted in the following. Denote with $\mathbf{n}$ the unit normal vector to the plane resolved in IFR. Then the vector from the contact point $P$ to the $\operatorname{COM}$ is $\rho=\rho \mathbf{n}$, and the rolling-without-slipping condition is

$$
\mathbf{0}=\dot{\mathbf{r}}+\widetilde{\rho} \omega=\dot{\mathbf{r}}+\widetilde{\rho} \Omega \dot{\varphi}
$$




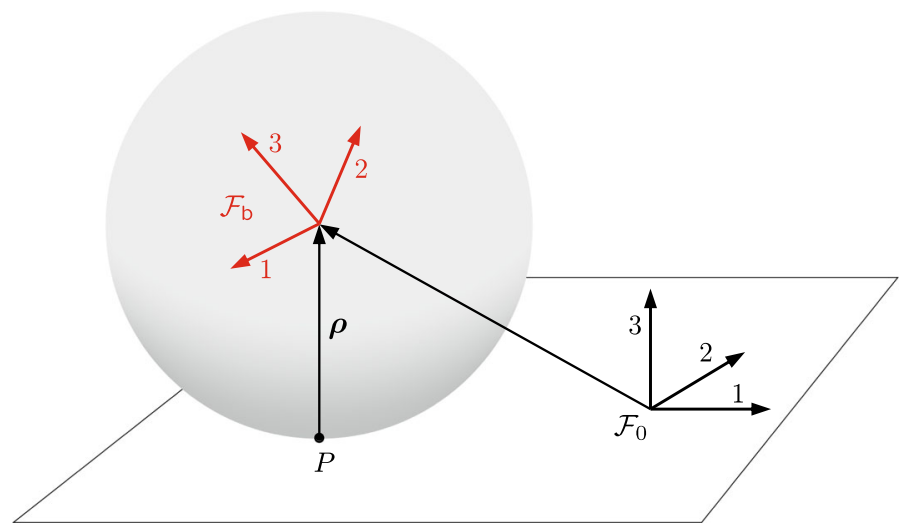

Fig. 1 Solid ball rolling on a horizontal plane

which yields a system of $\bar{m}=3$ Pfaffian constraints (20) on $\dot{\mathbf{q}}$, with

$$
u^{\alpha}:=\dot{r}_{\alpha}+\varepsilon_{\alpha j k} \rho_{j} \Omega_{k l} \dot{\varphi}^{l}, \quad \alpha=1,2,3
$$

The components of the angular velocity constitute $n-\bar{m}=3$ independent quasivelocities

$$
u^{I}:=\omega_{\bar{I}}, I=4,5,6
$$

Where the notation $\bar{I}:=I-3$ will be used. The constraints (126) along with $\omega_{\bar{I}}=$ $\Omega_{\bar{I} i} \dot{\varphi}^{i}$ give rise to the Pfaffian system (21) with matrix

$$
\mathbf{A}=\left(\begin{array}{cc}
\tilde{\rho} \boldsymbol{\Omega} & \mathbf{I} \\
\boldsymbol{\Omega} & \mathbf{0}
\end{array}\right)
$$

The solution (4) determines the $\dot{q}^{a}$ in terms of the Pfaffian forms $u^{a}$, where the inverse of $\mathbf{A}$ is

$$
\mathbf{B}=\left(\begin{array}{cc}
\mathbf{0} & \boldsymbol{\Psi} \\
\mathbf{I} & -\tilde{\rho}
\end{array}\right) .
$$

In components this is

$$
\begin{aligned}
& \dot{r}_{\alpha}=u^{\alpha}-\varepsilon_{\alpha j k} \rho_{j} \Omega_{k l} \dot{\varphi}^{l}, \quad \alpha=1,2,3 \\
& \dot{\varphi}^{i}=\Psi_{I}^{i} u^{I}, \quad I=4,5,6 .
\end{aligned}
$$

\subsubsection{Hamel Coefficients}

The Hamel equations (22) involve the coefficients $\gamma_{I J}^{a}, a=1, \ldots, 6 ; I, J=4,5,6$. First consider the case $a=K=4,5,6$. Since A does not depend on $\mathbf{r}$, it is $\frac{\partial A_{a b}}{\partial q^{c}} \equiv 0$ 
for $b, c=4,5,6$, and (23) yields

$$
\gamma_{I J}^{K}=\left(\frac{\partial \Omega_{\bar{K} i}}{\partial \varphi^{j}}-\frac{\partial \Omega_{\bar{K} j}}{\partial \varphi^{i}}\right) \Psi_{\bar{I}}^{i} \Psi_{\bar{J}}^{j} .
$$

In analogy to (42), using (154), the expression (132) is the definition of the Hamel coefficients (55) for the spatial angular velocity, i.e.

$$
\gamma_{I J}^{K}=-\varepsilon_{\bar{K}} \bar{I} \bar{J}, \quad I, J, K=4,5,6 .
$$

Noting that $\rho_{i}=$ const. in (126), the Hamel coefficients for $a=\alpha=1,2,3$ are

$$
\begin{aligned}
\gamma_{I J}^{\alpha} & =\varepsilon_{\alpha k l} \rho_{k}\left(\frac{\partial \Omega_{l i}}{\partial \varphi^{j}}-\frac{\partial \Omega_{l j}}{\partial \varphi^{i}}\right) \Psi_{\bar{I}}^{i} \Psi_{\bar{J}}^{j} \\
& =-\varepsilon_{\alpha k l} \rho_{k} \varepsilon_{l \bar{I} \bar{J}}, \quad \alpha=1,2,3 ; I, J=4,5,6 .
\end{aligned}
$$

\subsubsection{Hamel Equations}

With the assumption that the COM is at the center of the ball, and that it is rolling on a horizontal plane, its motion is not affected by gravity. The Lagrangian thus consists of the kinetic energy only. The kinetic energy of the unconstrained ball, in terms of the velocity $\dot{\mathbf{r}}$ of its $\mathrm{COM}$ and the angular velocity $\omega$, is

$$
T\left(q^{a}, \dot{q}^{a}\right)=\frac{1}{2} \omega_{i}(\dot{\varphi}){ }^{\mathrm{s}} \Theta_{i j}^{c}(\varphi) \omega_{j}(\dot{\varphi})+\frac{m}{2} \dot{r}_{i} \dot{r}_{i}
$$

where $m$ is its mass and ${ }^{\mathrm{s}} \boldsymbol{\Theta}^{\mathrm{C}}=\mathbf{R} \boldsymbol{\Theta}^{\mathrm{C}} \mathbf{R}^{T}$ the inertia tensor w.r.t. its COM, as in (114) with $\mathbf{d}=\mathbf{0}$, resolved in the IFR. Replacing $\dot{q}^{a}$ with (130) yields the kinetic energy in terms of $u^{a}$

$$
\mathcal{T}\left(q^{a}, u^{a}\right)=\frac{1}{2} u^{I}\left({ }^{s} \Theta_{\bar{I} \bar{J}}^{c}-m \varepsilon_{\bar{I} i \alpha} \varepsilon_{\alpha j \bar{J}} \rho_{i} \rho_{j}\right) u^{J}+\frac{m}{2} u^{\alpha} u^{\alpha}-m u^{\alpha} \varepsilon_{\alpha i \bar{I}} \rho_{i} u^{I}
$$

With the Hamel coefficients (133) and (135), the Hamel equations (22) in terms of $u^{a}$ are

$$
\frac{d}{d t} \frac{\partial \mathcal{T}}{\partial u^{I}}-\frac{\partial \mathcal{T}}{\partial \varphi^{i}} \Psi_{\bar{I}}^{i}-\frac{\partial \mathcal{T}}{\partial u^{\alpha}} \varepsilon_{\alpha k l} \rho_{k} \varepsilon_{l \bar{I} \bar{J}} u^{J}-\frac{\partial \mathcal{T}}{\partial u^{K}} \varepsilon_{\bar{K} \bar{I} \bar{J}} u^{J}=0, \quad I=4,5,6
$$

Using $\frac{d}{d t}\left({ }^{\mathrm{s}} \Theta_{i j}^{\mathrm{c}} \omega_{j}\right)={ }^{\mathrm{s}} \Theta_{i j}^{\mathrm{c}} \dot{\omega}_{j}+\varepsilon_{i k l} \omega_{k}{ }^{\mathrm{s}} \Theta_{l j}^{\mathrm{c}}$, the necessary partial derivatives are

$$
\begin{aligned}
\frac{d}{d t} \frac{\partial \mathcal{T}}{\partial u^{I}} & =\left({ }^{s} \Theta_{\bar{I} \bar{J}}^{c}-m \varepsilon_{\bar{I} i \alpha} \varepsilon_{\alpha j \bar{J}} \rho_{i} \rho_{j}\right) \dot{u}^{J}+\varepsilon_{\bar{I} \bar{J} i}{ }^{\mathrm{s}} \Theta_{i \bar{K}}^{\mathrm{c}} u^{K}, \frac{\partial \mathcal{T}}{\partial u^{\alpha}} \\
& =-m \varepsilon_{\alpha i \bar{I}} \rho_{i} u^{I}+m u^{\alpha}, \frac{\partial \mathcal{T}}{\partial \varphi^{i}} \Psi_{\bar{I}}^{i}=-\varepsilon_{\bar{I} \bar{J} i} u^{J \mathrm{~s}^{\mathrm{s}} \Theta_{i \bar{K}}^{\mathrm{c}} u^{K}}
\end{aligned}
$$


Inserted in (138), and setting $u^{\alpha}=0$, leads to

$$
\begin{aligned}
& \left({ }^{\mathrm{s}} \Theta_{\bar{I} \bar{J}}^{\mathrm{c}}-m \varepsilon_{\bar{I} i \alpha} \varepsilon_{\alpha j \bar{J}} \rho_{i} \rho_{j}\right) \dot{u}^{J}+\varepsilon_{\bar{I} \bar{J} i} u^{J \mathrm{~s}} \Theta_{i \bar{K}}^{\mathrm{c}} u^{K} \\
& \quad+m\left(\varepsilon_{\bar{K} i \alpha} \varepsilon_{\alpha j \bar{L}} \rho_{i} \rho_{r} \varepsilon_{\bar{K} \bar{I} \bar{J}}-m u^{K} \varepsilon_{\bar{K} i \alpha} \varepsilon_{\alpha r s} \rho_{i} \rho_{r} \varepsilon_{s \bar{I} \bar{J}}\right) u^{J}=0, \quad I=4,5,6 .
\end{aligned}
$$

The last term in (140) vanishes, and replacing $u^{I}=\omega_{\bar{I}}$, the Hamel equations of the rolling ball are

$$
{ }^{\mathrm{s}} \dot{L}_{i}^{\mathrm{P}}={ }^{\mathrm{s}} \Theta_{i j}^{\mathrm{P}} \dot{\omega}_{j}^{\mathrm{s}}+\varepsilon_{i j k} \omega_{j}^{\mathrm{s} \mathrm{s}} \Theta_{k l}^{\mathrm{c}} \omega_{l}^{\mathrm{s}}=0,
$$

where ${ }^{\mathrm{s}} \Theta_{i j}^{\mathrm{P}}:={ }^{\mathrm{s}} \Theta_{i j}^{\mathrm{c}}-m \varepsilon_{i r k} \varepsilon_{k s j} \rho_{r} \rho_{s}$ is the inertia tensor w.r.t. the contact point $P$ expressed in the IFR, and ${ }^{\mathrm{s}} L_{i}^{\mathrm{P}}:={ }^{\mathrm{s}} \Theta_{i j}^{\mathrm{P}} \omega_{j}$ is the angular momentum w.r.t. to $P$. Relation (141) is nothing but the condition for conservation of angular momentum. However, it is important to note that this is only true since the vector $\rho$ is constant. This does not hold if the COM is not at the geometric center. The vector form of (141) is, with ${ }^{\mathrm{s}} \boldsymbol{\Theta}^{\mathrm{P}}:={ }^{\mathrm{s}} \boldsymbol{\Theta}^{\mathrm{C}}-m \tilde{\boldsymbol{\rho}} \widetilde{\rho}$ and ${ }^{\mathrm{s}} \mathbf{L}^{\mathrm{P}}:={ }^{\mathrm{s}} \boldsymbol{\Theta}^{\mathrm{P}} \boldsymbol{\omega}^{\mathrm{s}}$,

$$
{ }^{\mathrm{S}} \dot{\mathbf{L}}^{\mathrm{P}}={ }^{\mathrm{s}} \boldsymbol{\Theta}^{\mathrm{P}} \dot{\boldsymbol{\omega}}^{\mathrm{S}}+\widetilde{\boldsymbol{\omega}}^{\mathrm{s} \mathrm{S}} \boldsymbol{\Theta}^{\mathrm{C}} \boldsymbol{\omega}^{\mathrm{S}}=\mathbf{0} .
$$

\section{Conclusion}

The classical problem of rigid body dynamics in terms of quasi-velocities has been addressed using the BH-equations and the original definition of the Hamel coefficients. The Newton-Euler equations are recovered and shown to be the identical to the Euler-Poincaré equations, if the body-fixed twists are used as quasi-velocities, and to the reduced Euler-Lagrange equations, if the spatial and hybrid representation of rigid body twists are used. In these cases the Hamel coefficients are identical to the structure constants of $S E(3)$ and $S O(3) \times \mathbb{R}^{3}$, respectively. For illustration purpose the $\mathrm{BH}$-equations of a rolling homogenous ball are derived to demonstrated the Hamel-equations for a constrained rigid body.

The novel contribution of this paper is the explicit derivation of the Hamel coefficients for the three most relevant representations of rigid body twists, without resorting to a description in terms of local coordinates, using their original definition. This shall complement the classical treatment of rigid body dynamics by means of the $\mathrm{BH}$ equations. The presented approach offers a direct link between the classical treatment and the modern Lie group methods for discrete mechanical systems evolving on a Lie group.

Finally it is to be mentioned that Hamel's variational approach to the dynamics of constrained rigid bodies is not only relevant for optimal control (Maruskin and Bloch 2007) but has led to advanced Lie group modeling and numerical integration schemes for robots and general multibody systems (Ball and Zenkov 2015; Borri et al. 2003; Brüls and Cardona 2010; Hairer et al. 2006; Müller 2018; Park and Chung 2005; Terze et al. 2014, 2015; Zenkov et al. 2012). 
Acknowledgements The author would like Dr. Abhinandan Jain from NASA Jet Propulsion Laboratory for pointing out the lack of a explicit coordinate-free derivation of the Hamel coefficients for the rigid body. The author acknowledges support by the LCM-K2 Center within the framework of the Austrian COMET-K2 program.

Funding Open access funding provided by Johannes Kepler University Linz.

Open Access This article is licensed under a Creative Commons Attribution 4.0 International License, which permits use, sharing, adaptation, distribution and reproduction in any medium or format, as long as you give appropriate credit to the original author(s) and the source, provide a link to the Creative Commons licence, and indicate if changes were made. The images or other third party material in this article are included in the article's Creative Commons licence, unless indicated otherwise in a credit line to the material. If material is not included in the article's Creative Commons licence and your intended use is not permitted by statutory regulation or exceeds the permitted use, you will need to obtain permission directly from the copyright holder. To view a copy of this licence, visit http://creativecommons.org/licenses/by/4.0/.

\section{A Rigid Body Motions}

The motion of a rigid body is represented by the motion of a body-fixed reference frame $\mathcal{F}_{\mathrm{b}}$, generally located at the body. The pose of the rigid body is thus described by the frame transformation from the body-fixed frame $\mathcal{F}_{\mathrm{b}}$ to an IFR $\mathcal{F}_{0}$. This frame transformation, and thus the pose of the body, is determined by the position vector $\mathbf{r} \in \mathbb{R}^{3}$ of the body-fixed frame measured and resolved in the IFR, along with the rotation matrix $\mathbf{R} \in S O$ (3) transforming coordinates of a vector resolved in a bodyfixed frame to those resolved in the IFR.

\section{A.1 Rotations: Lie Group SO(3)}

Let $\mathbf{R}=\left(R_{i j}\right) \in S O$ (3) be the rotation matrix transforming from body-fixed to IFR. An arbitrary parameterization in terms of three variables $\varphi=\left(\varphi^{1}, \varphi^{2}, \varphi^{3}\right)$ is assumed, i.e. $\mathbf{R}=\mathbf{R}(\varphi)$. Typical choices are Euler or Cardan angles, or the three components of the product of rotation axis and rotation angle.

The angular velocity vector in body-fixed representation, denoted $\omega^{\mathrm{b}}$ $=\left(\omega_{1}^{\mathrm{b}}, \omega_{2}^{\mathrm{b}}, \omega_{3}^{\mathrm{b}}\right)^{T}$, is defined via the skew-symmetric angular velocity tensor $\widetilde{\boldsymbol{\omega}}^{\mathrm{b}}=$ $\mathbf{R}^{T} \dot{\mathbf{R}}$. The latter is related to $\omega^{\mathrm{b}}$ by the correspondence

$$
\mathbf{x} \in \mathbb{R}^{3} \leftrightarrow \widetilde{\mathbf{x}} \in \operatorname{so}(3)
$$

where the components of $\widetilde{\mathbf{x}}=\left(x_{i j}\right) \in \operatorname{so}(3)$ are $x_{i j}=\varepsilon_{i k j} x_{k}$. The matrix (143) is frequently called the cross product matrix since $\widetilde{\mathbf{x}} \mathbf{y}=\mathbf{x} \times \mathbf{y}$. With the parameterization in terms of $\boldsymbol{\varphi}$, the body-fixed angular velocity tensor and vector are determined as

$$
\begin{aligned}
& \widetilde{\boldsymbol{\omega}}^{\mathrm{b}}=\mathbf{R}^{T} \dot{\mathbf{R}}=\mathbf{R}^{T} \frac{\partial \mathbf{R}}{\partial \varphi^{a}} \dot{\varphi}^{a}=\widetilde{\boldsymbol{\Omega}}_{a}^{\mathrm{b}} \dot{\varphi}^{a}, \quad \widetilde{\boldsymbol{\Omega}}_{a}^{\mathrm{b}}:=\mathbf{R}^{T} \frac{\partial \mathbf{R}}{\partial \varphi^{a}} \\
& \boldsymbol{\omega}^{\mathrm{b}}=\sum_{a} \boldsymbol{\Omega}_{a}^{\mathrm{b}} \dot{\varphi}^{a}=\boldsymbol{\Omega}^{\mathrm{b}} \dot{\boldsymbol{\varphi}}, \quad \boldsymbol{\Omega}^{\mathrm{b}}=\left(\boldsymbol{\Omega}_{1}^{\mathrm{b}}, \boldsymbol{\Omega}_{2}^{\mathrm{b}}, \boldsymbol{\Omega}_{3}^{\mathrm{b}}\right)
\end{aligned}
$$


where $\boldsymbol{\Omega}_{a}^{\mathrm{b}}$ is the $a$-th column of $\boldsymbol{\Omega}^{\mathrm{b}}=\left(\Omega_{i a}^{\mathrm{b}}\right)$. Written in component form, these relations are

$$
\begin{aligned}
\omega_{i j}^{\mathrm{b}} & =\varepsilon_{i k j} \omega_{k}^{\mathrm{b}}=R_{k i} \frac{\partial R_{k j}}{\partial \varphi_{a}} \dot{\varphi}^{a} \\
& =\varepsilon_{i k j} \Omega_{k a}^{\mathrm{b}} \dot{\varphi}^{a} \\
\omega_{i}^{\mathrm{b}} & =\Omega_{i a}^{\mathrm{b}} \dot{\varphi}^{a}
\end{aligned}
$$

From (145) and (146) follows

$$
\begin{aligned}
\varepsilon_{i k j} \Omega_{k a}^{\mathrm{b}} & =\frac{\partial \omega_{i j}^{\mathrm{b}}}{\partial \dot{\varphi}^{a}} \\
\boldsymbol{\Omega}_{a}^{\mathrm{b}} & =\frac{\partial \omega^{\mathrm{b}}}{\partial \dot{\varphi}^{a}}, \Omega_{i a}^{\mathrm{b}}=\frac{\partial \omega_{i}^{\mathrm{b}}}{\partial \dot{\varphi}^{a}}
\end{aligned}
$$

The inverse relation to (148) is

$$
\dot{\varphi}^{a}=\Psi_{i}^{a, \mathrm{~b}} \omega_{i}^{\mathrm{b}} \text { with } \quad \Psi^{\mathrm{b}}:=\left(\boldsymbol{\Omega}^{\mathrm{b}}\right)^{-1} .
$$

Combining this with (146) yields

$$
R_{k i} \omega_{i j}^{\mathrm{b}}=\frac{\partial R_{k j}}{\partial \varphi^{a}} \Psi_{r}^{a, \mathrm{~b}} \omega_{r}^{\mathrm{b}}, \quad R_{k r} \varepsilon_{r i j}=\frac{\partial R_{k j}}{\partial \varphi^{a}} \Psi_{i}^{a, \mathrm{~b}} .
$$

In spatial representation the angular velocity $\omega^{\mathrm{s}}=\left(\omega_{1}^{\mathrm{s}}, \omega_{2}^{\mathrm{s}}, \omega_{3}^{\mathrm{s}}\right)^{T}$ is defined by $\widetilde{\boldsymbol{\omega}}^{\mathrm{s}}=\dot{\mathbf{R}} \mathbf{R}^{T}$. In analogy to (144), (148), it is expressed using the parameterization as

$$
\begin{aligned}
& \widetilde{\boldsymbol{\omega}}^{\mathrm{s}}=\dot{\mathbf{R}} \mathbf{R}^{T}=\frac{\partial \mathbf{R}}{\partial \varphi^{a}} \mathbf{R}^{T} \dot{\varphi}^{a}=\widetilde{\boldsymbol{\Omega}}_{a}^{\mathrm{s}} \dot{\varphi}^{a}, \quad \widetilde{\boldsymbol{\Omega}}_{a}^{\mathrm{s}}:=\frac{\partial \mathbf{R}}{\partial \varphi^{a}} \mathbf{R}^{T} \\
& \boldsymbol{\omega}^{\mathrm{s}}=\sum_{a} \boldsymbol{\Omega}_{a}^{\mathrm{s}} \dot{\varphi}^{a}=\boldsymbol{\Omega}^{\mathrm{s}} \dot{\boldsymbol{\varphi}}, \quad \boldsymbol{\Omega}^{\mathrm{s}}=\left(\boldsymbol{\Omega}_{1}^{\mathrm{s}}, \boldsymbol{\Omega}_{2}^{\mathrm{s}}, \boldsymbol{\Omega}_{3}^{\mathrm{s}}\right) .
\end{aligned}
$$

The inverse of this relation is $\dot{\varphi}^{a}=\Psi_{i}^{a, \mathrm{~s}} \omega_{i}^{\mathrm{s}}$, with $\Psi^{\mathrm{s}}:=\left(\boldsymbol{\Omega}^{\mathrm{s}}\right)^{-1}$.

With the isomorphism (143), the Lie bracket on so (3) can be expressed in matrix or vector representation, respectively,

$$
[\widetilde{\mathbf{x}}, \widetilde{\mathbf{y}}]=\widetilde{\mathbf{x}} \widetilde{\mathbf{y}}-\widetilde{\mathbf{y}} \widetilde{\mathbf{x}}, \quad[\mathbf{x}, \mathbf{y}]=\widetilde{\mathbf{x}} \mathbf{y}=\operatorname{ad}_{\mathbf{x}} \mathbf{y}
$$

with $\mathbf{x}, \mathbf{y} \in \mathbb{R}^{3}$, where $\mathbf{a d}_{\mathbf{x}}=\widetilde{\mathbf{x}}$. The respective components are

$$
[\widetilde{\mathbf{x}}, \widetilde{\mathbf{y}}]_{i j}=\varepsilon_{i k j} \varepsilon_{k r s} x_{r} y_{s}, \quad[\mathbf{x}, \mathbf{y}]_{i}=\varepsilon_{i j k} x_{j} y_{k}
$$

which follows from the identity $\varepsilon_{k i j} \varepsilon_{k r s}=\delta_{i r} \delta_{j s}-\delta_{i s} \delta_{j r}$. That is, the structure constants of $S O(3)$ are given by the Levi-Civita symbol $\varepsilon_{i j k}$. 


\section{A.2 Spatial Rigid Body Motions}

\section{A.2.1 Rigid Body Motions as Frame Transformations: Lie Group SE(3)}

The motion of a rigid body is described by the motion of a body-fixed frame $\mathcal{F}$. The configuration (pose, posture) of the body is described by a frame transformation from body-fixed to inertial frame. This is described by the rotation matrix $\mathbf{R} \in S O$ (3), transforming coordinates of a vector when expressed in the body-fixed frame to those when expressed in inertia frame, and the position vector $\mathbf{r} \in \mathbb{R}^{3}$ of the origin of the body-fixed frame measured in the IFR. Frame transformations form the Lie group $S E$ (3), and are denoted as $C=(\mathbf{R}, \mathbf{r}) \in S E(3)$, and frequently represented by 'homogenous transformation' matrices

$$
\mathbf{C}=\left(\begin{array}{ll}
\mathbf{R} & \mathbf{r} \\
\mathbf{0} & 1
\end{array}\right) \in S E(3)
$$

that transform point coordinates measured in a body-fixed frame to those measured in the IFR. For details on $S E$ (3) the reader is referred to Selig (2005).

The transition from one configuration to another is described by a repeated frame transformation, and achieved by the group multiplication $C_{1} \cdot C_{2}=$ $\left(\mathbf{R}_{1} \mathbf{R}_{2}, \mathbf{r}_{1}+\mathbf{R}_{1} \mathbf{r}_{2}\right)$, or in matrix form $\mathbf{C}_{1} \mathbf{C}_{2}$. This multiplication reveals that $S E(3)=$ $S O(3) \ltimes \mathbb{R}^{3}$ is the semi-direct product of the rotation group and the translation group.

The body-fixed twist of the rigid body is defined as the left-invariant vector field $T_{C} L_{C^{-1}} \dot{C}=\left(\widetilde{\omega}^{\mathrm{b}}, \mathbf{v}^{\mathrm{b}}\right)$. This is usually expressed in matrix form as

$$
\mathbf{C}^{-1} \dot{\mathbf{C}}=\left(\begin{array}{cc}
\widetilde{\boldsymbol{\omega}}^{\mathrm{b}} & \mathbf{v}^{\mathrm{b}} \\
\mathbf{0} & 0
\end{array}\right)=\widehat{\mathbf{V}}^{\mathrm{b}} \in \operatorname{se}(3)
$$

with the body-fixed angular velocity $\omega^{\mathrm{b}} \in \mathbb{R}^{3}$ and translational velocity $\mathbf{v}^{\mathrm{b}}=\mathbf{R}^{T} \dot{\mathbf{r}} \in$ $\mathbb{R}^{3}$. This defines $\mathbf{V}^{\mathrm{b}} \in \mathbb{R}^{6}$ according to

$$
\mathbf{V}^{\mathrm{b}}=\left(\begin{array}{c}
\omega^{\mathrm{b}} \\
\mathbf{v}^{\mathrm{b}}
\end{array}\right) \in \mathbb{R}^{6} \leftrightarrow \widehat{\mathbf{V}}^{\mathrm{b}}=\left(\begin{array}{cc}
\widetilde{\omega}^{\mathrm{b}} & \mathbf{v}^{\mathrm{b}} \\
\mathbf{0} & 0
\end{array}\right) \in s e(3) .
$$

The spatial twist of the rigid body is defined as the right-invariant vector fields $T_{C} R_{C^{-1}} \dot{C}=\left(\widetilde{\omega}^{\mathrm{s}}, \mathbf{v}^{\mathrm{s}}\right)$, and in matrix form

$$
\dot{\mathbf{C}} \mathbf{C}^{-1}=\left(\begin{array}{cc}
\widetilde{\omega}^{s} & \mathbf{v}^{s} \\
\mathbf{0} & 0
\end{array}\right)=\widehat{\mathbf{V}}^{s} \in s e(3)
$$

where $\mathbf{v}^{\mathrm{s}}=\dot{\mathbf{r}}-\widetilde{\boldsymbol{\omega}}^{\mathrm{s}} \mathbf{r} \in \mathbb{R}^{3}$ is the velocity of the body-fixed point momentarily coinciding with the origin of the IFR. This is also called the fixed pole formulation (Bottasso and Borri 1998). The corresponding spatial twist vector is $\mathbf{V}^{\mathrm{b}}=\left(\boldsymbol{\omega}^{\mathrm{s}}, \mathbf{v}^{\mathrm{s}}\right)$.

It will be necessary to express the relation (159) of matrix $\widehat{\mathbf{V}} \in \operatorname{se}(3)$ and vector $\mathbf{V} \in \mathbb{R}^{6}$ in components. Notice again that the basis on $s e(3)$ is deduced from an orthogonal Cartesian frame. To this end, in the following, Greek letters run over 
the range $1, \ldots, 4$, while italic letters run over $1, \ldots, 6$. The components of $\widehat{\mathbf{V}}=$ $\left(V_{\alpha \beta}\right) \in \operatorname{se}(3)$ are $V_{\alpha \beta}=h_{\alpha i \beta} V_{i}, i=1, \ldots, 6, \alpha, \beta=1, \ldots, 4$, where the constant coefficients

$$
h_{\alpha i \beta}= \begin{cases}\varepsilon_{\alpha i \beta}, & \alpha, \beta, i=1,2,3 \\ \delta_{\alpha \bar{i} 4}, & \alpha=1,2,3 ; \beta=4 ; i=4,5,6 \\ 0, & \text { otherwise }\end{cases}
$$

assign the six components of $\mathbf{V} \in \mathbb{R}^{6}$ to the six nonzero components of $\widehat{\mathbf{V}} \in$ se (3), similarly to the Levi-Civita symbol in (143) and (146). Here the notation $\bar{i}=i-3$ has been used.

The Lie bracket on se (3) can be expressed in matrix and vector representation, respectively, as

$$
\begin{aligned}
{[\widehat{\mathbf{X}}, \widehat{\mathbf{Y}}] } & =\widehat{\mathbf{X}} \widehat{\mathbf{Y}}-\widehat{\mathbf{Y}} \widehat{\mathbf{X}}=\left(\begin{array}{cc}
\widetilde{\omega}_{X} \widetilde{\boldsymbol{\omega}}_{Y}-\widetilde{\boldsymbol{\omega}}_{Y} \widetilde{\boldsymbol{\omega}}_{X} \widetilde{\boldsymbol{\eta}}_{X} \boldsymbol{\omega}_{Y}+\widetilde{\boldsymbol{\omega}}_{X} \boldsymbol{\eta}_{Y} \\
\mathbf{0} & 0
\end{array}\right), \quad[\mathbf{X}, \mathbf{Y}]=\operatorname{ad}_{\mathbf{X}} \mathbf{Y} \\
& =\left(\begin{array}{c}
\widetilde{\boldsymbol{\omega}}_{X} \boldsymbol{\omega}_{Y} \\
\widetilde{\boldsymbol{\omega}}_{X} \boldsymbol{\eta}_{Y}+\widetilde{\boldsymbol{\eta}}_{X} \boldsymbol{\omega}_{Y}
\end{array}\right)
\end{aligned}
$$

with matrix

$$
\operatorname{ad}_{\mathbf{X}}=\left(\begin{array}{cc}
\widetilde{\omega} & \mathbf{0} \\
\widetilde{\eta} & \widetilde{\omega}
\end{array}\right)
$$

The correspondence (159), i.e. the isomorphism $\widehat{[\mathbf{X}, \mathbf{Y}]}=[\widehat{\mathbf{X}}, \widehat{\mathbf{Y}}]$, yields the components of the Lie bracket in matrix representation and vector representation of se (3), respectively, as

$$
[\widehat{\mathbf{X}}, \widehat{\mathbf{Y}}]_{\alpha \beta}=h_{\alpha i \beta} c_{i j k} X_{j} Y_{k}, \quad[\mathbf{X}, \mathbf{Y}]_{i}=c_{i j k} X_{j} Y_{k}
$$

where $c_{i j k}$ are the structure constants of $s e(3)$,

$$
c_{i j k}= \begin{cases}\varepsilon_{i j k}, & \alpha, \beta, i=1,2,3 \\ \varepsilon_{i j \bar{k}}^{-}, & i, k=4,5,6 ; j=1,2,3 \\ \varepsilon_{\bar{i} \bar{j} k}, & i, j=4,5,6 ; k=1,2,3 \\ 0, & \text { otherwise. }\end{cases}
$$

It is apparent from the structure constants that $S E(3)$ is the semi-direct product. The correspondence (164) of the components is established by the identity

$$
h_{\alpha i \rho} h_{\rho j \beta}-h_{\alpha j \rho} h_{\rho i \beta}=h_{\alpha k \beta} c_{k i j} .
$$

The rigid body transformation is parameterized in terms of canonical coordinates $\mathbf{q}=\left(q^{1}, \ldots, q^{6}\right)$ on $S E(3)$, e.g. screw coordinates or three angles together with the three components of the position vector. The body-fixed twist is then expressed in terms of their time derivatives as

$$
\widehat{\mathbf{V}}^{\mathrm{b}}=\mathbf{C}^{-1} \dot{\mathbf{C}}=\sum_{a} \mathbf{C}^{-1} \frac{\partial \mathbf{C}}{\partial q^{a}} \dot{q}^{a}=\sum_{a} \widehat{\mathbf{J}}_{a} \dot{q}^{a}, \quad \widehat{\mathbf{J}}_{a}:=\mathbf{C}^{-1} \frac{\partial \mathbf{C}}{\partial q^{a}}
$$




$$
\mathbf{V}^{\mathrm{b}}=\sum_{a} \mathbf{J}_{a} \dot{q}^{a}=\mathbf{J} \dot{\mathbf{q}}, \quad \mathbf{J}=\left(\mathbf{J}_{1}, \ldots, \mathbf{J}_{6}\right)
$$

where $\mathbf{J}_{a}$ is the $a$-th column of $\mathbf{J}=\left(J_{i a}\right)$. Denote the inverse of the transformation matrix (157) with $\mathbf{D}:=\mathbf{C}^{-1}$. Then, with $\mathbf{C}=\left(C_{\alpha \beta}\right), \mathbf{D}=\left(D_{\alpha \beta}\right)$ and $\mathbf{V}=\left(V_{i}\right)$, $\widehat{\mathbf{V}}=\left(V_{\alpha \beta}\right)$, the relations (167) and (168) are in component form

$$
\begin{aligned}
V_{i} & =J_{i a} \dot{q}^{a} \\
V_{\alpha \beta} & =h_{\alpha i \beta} V_{i}=h_{\alpha i \beta} J_{i a} \dot{q}^{a}=D_{\alpha \sigma} \frac{\partial C_{\sigma \beta}}{\partial q^{a}} \dot{q}^{a}=-\frac{\partial D_{\alpha \sigma}}{\partial q^{a}} C_{\sigma \beta} \dot{q}^{a} .
\end{aligned}
$$

These relations immediately give rise to the following expression for the components of $\mathbf{J}=\left(J_{i a}\right)$

$$
\begin{aligned}
\mathbf{J}_{a} & =\frac{\partial \mathbf{V}}{\partial \dot{q}^{a}}, \quad J_{i a}=\frac{\partial V_{i}}{\partial \dot{q}^{a}} \\
h_{\alpha i \beta} J_{i a} & =\frac{\partial V_{\alpha \beta}}{\partial \dot{q}^{a}} .
\end{aligned}
$$

The inverse relation to (169) is

$$
\dot{q}^{a}=G_{i}^{a} V_{i} \text { with } \mathbf{G}=\mathbf{J}^{-1} .
$$

Inserting (173) in (170) yields

$$
\frac{\partial C_{\sigma \beta}}{\partial q^{a}} G_{i}^{a}=C_{\sigma \alpha} h_{\alpha i \beta}, \quad \frac{\partial D_{\alpha \sigma}}{\partial q^{a}} G_{i}^{a}=-h_{\alpha i \beta} D_{\beta \sigma} .
$$

\section{A.2.2 Rigid Body Motions as Decoupled Rotations and Translations: Lie Group SO (3) $\times \mathbb{R}^{3}$}

It is a fundamental property of rigid body motions, which is clear from the frame transformation (157) of point coordinates, that rotations and translations are coupled, but independent. Nevertheless, in many formulations in computational dynamics and multibody system dynamics, translations and rotations are treated decoupled (Shabana 2013; Brüls and Cardona 2010). This is motivated by using the NE equations relative to the COM (i.e. $\mathbf{d}=\mathbf{0}$ in (124)). The underlying geometric model is the direct product $S O$ (3) $\times \mathbb{R}^{3}$ with group operation $C_{1} \cdot C_{2}=\left(\mathbf{R}_{1} \mathbf{R}_{2}, \mathbf{r}_{1}+\mathbf{r}_{2}\right)$. This does clearly not describe general rigid body motions.

Let $\omega^{5}$ be the spatial angular velocity, and $\dot{\mathbf{r}}$ be the velocity of the origin of a body-fixed frame represented in the IFR. Then,

$$
\mathbf{V}^{\mathrm{h}}=\left(\omega^{\mathrm{s}}, \dot{\mathbf{r}}\right)
$$

is called the hybrid form of the body twist since it is the twist measured at the origin of the body-fixed frame but resolved in the IFR (Murray et al. 1994). The hybrid twist can be defined as the right-invariant vector field $T_{C} R_{C^{-1}} \dot{C}=\left(\widetilde{\boldsymbol{\omega}}^{\mathrm{s}}, \dot{\mathbf{r}}\right)$, for $C \in S O(3) \times \mathbb{R}^{3}$. 


\section{B List of Symbols}

$$
\begin{aligned}
& \mathcal{F}_{0} \\
& \mathcal{F}_{\mathrm{b}} \\
& \mathcal{F}_{\text {c }} \\
& \mathbf{R}=\left(R_{i j}\right) \in S O \text { (3) } \\
& \mathbf{r}=\left(r_{i}\right) \in \mathbb{R}^{3} \\
& \mathbf{C}=\left(C_{\alpha \beta}\right) \in S E(3) \\
& \omega^{\mathrm{b}}=\left(\omega_{i}^{\mathrm{b}}\right) \in \mathbb{R}^{3} \\
& \omega^{\mathrm{s}}=\left(\omega_{i}^{\mathrm{s}}\right) \in \mathbb{R}^{3} \\
& \mathbf{v}^{\mathrm{b}}=\left(v_{i}^{\mathrm{b}}\right) \in \mathbb{R}^{3} \\
& \mathbf{v}^{\mathrm{s}}=\left(v_{i}^{\mathrm{s}}\right) \in \mathbb{R}^{3} \\
& \widetilde{\boldsymbol{\omega}}=\left(\omega_{i j}\right) \in \operatorname{so}(3) \\
& \mathbf{V}^{\mathrm{b}}=\left(V_{i}^{\mathrm{b}}\right) \in \mathbb{R}^{6} \\
& \mathbf{V}^{s}=\left(V_{i}^{s}\right) \in \mathbb{R}^{6} \\
& \mathbf{V}^{\mathrm{h}}=\left(V_{i}^{\mathrm{h}}\right) \in \mathbb{R}^{6} \\
& \widehat{\mathbf{V}}=\left(V_{\alpha \beta}\right) \in s e(3) \\
& \boldsymbol{\theta}^{\mathrm{s}}=\left(\theta_{i}^{\mathrm{s}}\right) \\
& \boldsymbol{\theta}^{\mathrm{b}}=\left(\theta_{i}^{\mathrm{b}}\right) \\
& \rho^{\mathrm{s}}=\left(\rho_{i}^{\mathrm{s}}\right) \\
& \rho^{\mathrm{b}}=\left(\rho_{i}^{\mathrm{b}}\right)
\end{aligned}
$$$$
\boldsymbol{\Theta}^{\mathrm{b}}=\left(\Theta_{i j}^{\mathrm{b}}\right)
$$$$
\boldsymbol{\Theta}^{\mathrm{s}}=\left(\Theta_{i j}^{\mathrm{s}}\right)
$$$$
{ }^{\mathrm{s}} \Theta^{\mathrm{b}}=\left({ }^{\mathrm{s}} \Theta_{i j}^{\mathrm{b}}\right)
$$$$
{ }^{\mathrm{s}} \Theta^{\mathrm{c}}=\left({ }^{\mathrm{s}} \Theta_{i j}^{\mathrm{c}}\right)
$$$$
q^{a}
$$

Spatial inertial frame (IFR)

Body-fixed reference frame at arbitrary location Body-fixed reference frame at COM

Rotation matrix transforming coordinates of a vector resolved in body frame to those when resolved in spatial inertial frame

Position vector of the origin of the body-fixed frame expressed in IFR

Homogenous transformation matrix from body-fixed to inertial frame

Coordinate vector of angular of $\mathcal{F}_{\mathrm{b}}$ relative to $\mathcal{F}_{0}$ resolved in $\mathcal{F}_{\mathrm{b}}$ (body-fixed representation)

Coordinate vector of angular of $\mathcal{F}_{\mathrm{b}}$ relative to $\mathcal{F}_{0}$ resolved in $\mathcal{F}_{0}$ (spatial representation)

Velocity of origin of $\mathcal{F}_{\mathrm{b}}$ measure and resolved in $\mathcal{F}_{0}$

Velocity of the point of a body coinciding with the origin of $\mathcal{F}_{0}$ measure and resolved in $\mathcal{F}_{0}$

Skew symmetric matrix associated to vector $\omega \in \mathbb{R}^{3}$, where $\omega_{i j}:=\varepsilon_{i k j} \omega_{k}$

Twist vector defined as $\mathbf{V}^{\mathrm{b}}=\left(\boldsymbol{\omega}^{\mathrm{b}}, \mathbf{v}^{\mathrm{b}}\right)$ in body-fixed representation

Twist vector defined as $\mathbf{V}^{s}=\left(\omega^{s}, \mathbf{v}^{s}\right)$ in spatial representation

Twist vector defined as $\mathbf{V}^{\mathrm{h}}=\left(\boldsymbol{\omega}^{\mathrm{s}}, \dot{\mathbf{r}}\right)$ in hybrid representation

Representation of the twist by a $4 \times 4$-matrix (twist tensor), where $V_{\alpha \beta}:=h_{\alpha k \beta} V_{k}$ with $h_{\alpha k \beta}$ in (161)

Quasi-coordinates for the orientation of the body-fixed frame

Resolved in spatial inertial frame and body-fixed frame, respectively

Quasi-coordinates for the position of the origin of bodyfixed frame

Resolved in spatial inertial frame and body-fixed frame, respectively These are summarized as $\pi^{\mathrm{b}}=\left(\boldsymbol{\theta}^{\mathrm{b}}, \rho^{\mathrm{b}}\right)$ and $\pi^{\mathrm{s}}=\left(\theta^{\mathrm{s}}, \rho^{\mathrm{s}}\right)$.

Inertia tensor w.r.t. to the body-fixed frame

Inertia tensor w.r.t. to the body-fixed point coinciding with IFR origin resolved in IFR

Inertia tensor w.r.t. origin of body-fixed frame resolved in IFR

Inertia tensor w.r.t. COM resolved in IFR

Local coordinates, $a=1, \ldots, n$ 


$\begin{array}{ll}\pi^{a} & \text { Quasi-coordinates } \\ u^{a} & \text { Quasi-velocity coordinates } \\ \boldsymbol{\Omega}^{\mathrm{b}}=\left(\Omega_{i a}^{\mathrm{b}}\right) & \text { Coefficients of the kinematic relation } \omega_{i}^{\mathrm{b}}=\Omega_{i a}^{\mathrm{b}} \dot{\varphi}^{a} \text { (super- } \\ & \text { script s for spatial representation) } \\ \boldsymbol{\Psi}^{\mathrm{b}}=\left(\Psi_{i}^{a, \mathrm{~b}}\right) & \text { Coefficients of the inverse kinematic relation } \dot{\varphi}^{a}=\Psi_{i}^{a, \mathrm{~b}} \omega_{i}^{\mathrm{b}} \\ \mathbf{J}=\left(J_{i a}\right) & \text { Coefficients of the kinematic relation } V_{i}=J_{i a} \dot{q}^{a} \\ \mathbf{G}=\left(G_{i}^{a}\right) & \text { Coefficients of the inverse kinematic relation } \dot{q}^{a}=G_{i}^{a} V_{i} \\ n & \text { Number of (generalized) coordinates } \\ \bar{m} & \text { Number of Pfaffian constraints } \\ a, b, c, \ldots & \text { Indices running over all coordinates: } a=1, \ldots, n \\ I, K, L, \ldots & \text { Indices of independent velocity coordinates: } I=\bar{m}+ \\ \alpha, \beta, \gamma, \ldots & 1, \ldots, n \\ & \text { Indices of constraints equations and of dependent velocity } \\ \bar{I} & \text { coordinates: } \alpha=1, \ldots, \bar{m} \text { indices of an } s e(3) \text {-tensor: } \alpha= \\ \gamma_{i j}^{k} & 1, \ldots, 4 \\ \gamma & \text { Shifted index } \bar{I}:=I-3 \\ S E(3)=S O(3) \ltimes \mathbb{R}^{3} & \text { Hamel coefficients of a rigid body } \\ S O(3) & \text { Special Euclidean group (rigid body motion group) } \\ \mathrm{b} & \text { Special orthogonal group (rotation group) } \\ \mathrm{s} & \text { Indicates body-fixed representation (these are not indices) } \\ \mathrm{h} & \text { Indicates spatial representation } \\ & \text { Indicates hybrid representation }\end{array}$

\title{
C List of Abbreviations
}

\author{
IFR Inertial Frame \\ COM Center Of Mass \\ BH Boltzmann-Hamel
}

\section{References}

Ball, K.R., Zenkov, D.V.: Hamel's formalism and variational integrators. In: Chang, D.E., et al. (eds.) Geometry, Mechanics, and Dynamics, Fields Institute Communications, vol. 7, pp. 477-506 (2015)

Bloch, A.M., Marsden, J.E., Zenkov, D.V.: Nonholonomic dynamics. Notices AMS 52(3), 324-333 (2005)

Bloch, A.M., Marsden, J.E., Zenkov, D.V.: Quasivelocities and symmetries in nonholonomic systems. Dyn. Syst. Int. J. 24, 187-222 (2009)

Boltzmann, L.: Über die Form der Lagrange'schen Gleichungen für nicht holonome generalisierte Coordinaten, Sitzungsberichte der Kaiserlichen Akademie der Wissenschaften in Wien, No. 111, pp. 1603-1614 (1902). Published as F. Hasenöhrl (ed.): Wissenschaftliche Abhandlungen, by Ludwig Boltzmann, Friedrich Hasenöhrl, Cambridge University Press, 2012

Boltzmann, L.: Vorlesungen über die Prinzipe der Mechanik. Verlag von Johann Ambrosius Barth, Leipzig, II. Teil (1904)

Borri, M., Bottasso, C.L., Trainelli, L.: Integration of elastic multibody systems by invariant conserving/dissipating algorithms-part II: numerical schemes and applications. Comput. Methods Appl. Mech. Eng. 190(29/30), 3701-3733 (2001) 
Borri, M., Bottasso, C.L., Trainelli, L.: An invariant-preserving approach to robust finite-element multibody simulation. Zeitschrift für Angewandte Mathematik und Mechanik 83(10), 663-676 (2003)

Bottasso, C.L., Borri, M.: Integrating finite rotations. Comput. Methods Appl. Mech. Eng. 164, 307-331 (1998)

Brüls, O., Cardona, A.: On the use of Lie group time integrators in multibody dynamics. J. Comput. Nonlinear Dyn. 5(3) (2010)

Bullo, F., Lewis, A.D.: Geometric Control of Mechanical Systems. Springer, New York (2005)

Chaplygin, S.A.: On the motion of a heavy body of revolution on a horizontal plane. In: Proc. of the Physical Sciences' Section of the Society of Amateurs of Natural Sciences, vol. 9, pp. 10-16 (1897a). Reproduced in: Selected Works on Mechanics and Mathematics. Gostehteorizdat, Moscow, 1954, pp. 413-425. (in Russian) English translation.: Regular and Chaotic Dynamics, vol. 7, no. 2, pp. 119-130 (2002) https://doi.org/10.1070/RD2002v007n02ABEH000199

Chaplygin, S.A.: On some generalization of the area theorem with applications to the problem of rolling balls. Matematicheskii Sbornik 20 (1897b). (in Russian). English translation: Regular and Chaotic Dynamics, Vol. 17, No. 2, pp. 199-217 (2012) https://doi.org/10.1134/S1560354712020086

Chaplygin, S.A.: On the theory of motion of nonholonomic systems, the reducing-multiplier theorem. Matematicheskii Sbornik 28(1) (1911). (in Russian). English translation: Regular and Chaotic Dynamics 13, 369-376 (2008). https://doi.org/10.1134/S1560354708040102

Chaplygin, S.A.: On a ball's rolling on a horizontal plane. Matematicheskii Sbornik 24 (1903). (in Russian). English translation: Regular and Chaotic Dynamics, Vol. 17, No. 2, pp. 131-148 (2012) https://doi. org/10.1070/RD2002v007n02ABEH000200

Frobenius, F.G.: Über das Pfaffsche Problem. J. für reine und angewandte Mathematik 82, 230-315 (1875)

Guen, F.: On the equations of motion of nonholonomic mechanical systems in Poincaré-Chetaev variables. PMM 32(5), 864-814 (1968)

Hairer, E., Lubich, C., Wanner, G.: Geometric Numerical Integration. Springer, Berlin (2006)

Hamel, G.: Die Lagrange-Eulersche Gleichungen der Mechanik. Z. Math. Phys. 50(1), 1-57 (1904a)

Hamel, G.: Über die virtuellen Verschiebungen in der Mechanik, Math. Ann. Vol. LIX, pp. 416-434 (1904b)

Hamel, G.: Über nichtholonome Systeme. Math. Ann. 92, 33-41 (1924)

Hamel, G.: Theoretische Mechanik. Springer, Berlin (1949)

Hertz, H.: Die Prinzipien der Mechanik in neuem Zusammenhange dargestellt, (1894) (Ges. Werke. Bd. III. Leipzig. 1910)

Holm, D.: Geometric Mechanics Part II: Rotating, Translating and Rolling, 2nd edn. Imperial College Press, London (2011)

Hurtado, J.E., Sinclair, A.J.: Hamel coefficients for the rotational motion of an N-dimensional rigid body. Proc. R. Soc. Math. Phys. Eng. Sci. 460(2052), 3613-3630 (2004)

Lurie, I.A.: Analytical Mechanics. Springer, Berlin (2002)

Maisser, P.: Differential-geometric methods in multibody dynamics. Nonlinear Anal. Theory Methods Appl. 30(8), 5127-5133 (1997)

Maißer, P., Enge, O., Freudenberg, G., Kielau, G.: Electromechanical interactions in multibody systems containing electromechanical drives. Multibody Syst. Dyn. 1, 281-302 (1997)

Marsden, J., Ratiu, T.S.: Introduction to Mechanics and Symmetry: A Basic Exposition of Classical Mechanical Systems. Springer, Berlin (2003)

Marsden, J.E., Scheurle, J.: The reduced Euler-Lagrange equations. Fields Institute Commun. 1, 139-164 (1993)

Maruskin, J.M., Bloch, M.: The Boltzmann-Hamel equations for optimal control. In: 46th IEEE Conf. Dec. Contr., New Orleans, USA, Dec. 12-14 2007

Müller, A.: A note on the motion representation and configuration update in time stepping schemes for the constrained rigid body. BIT Numer. Math. 56(3) (2016)

Müller, A.: Screw and Lie group theory in multibody dynamics-recursive algorithms and equations of motion of tree-topology systems. Multibody Sys. Dyn. 42(2), 219-248 (2018)

Müller, A., Terze, Z.: The significance of the configuration space Lie group for the constraint satisfaction in numerical time integration of multibody systems. Mech. Mach. Theory 82, 173-202 (2014)

Murray, R.M., Li, Z., Sastry, S.S.: A Mathematical Introduction to Robotic Manipulation. CRC Press, Boca Raton (1994)

Neimark, J.I., Fufaev, N.A.: Dynamics of Nonholonomic Systems. Translations of Mathematical Monographs, vol. 33. AMS, Providence (1972)

Papastavridis, J.G.: Tensor Calculus and Analytical Dynamics. CRC Press, Boca Raton (1999) 
Papastavridis, J.G.: Analytical Mechanics. Oxford University Press, Oxford (2002)

Park, J., Chung, W.K.: Geometric integration on Euclidean group with application to articulated multibody systems. IEEE Trans. Robotics Automat. 21(5), 850-863 (2005)

Poincaré, H.: Les méthodes nouvelles de la m écanique céleste (1892)

Selig, J.: Geometric Fundamentals of Robotics. Springer, New York (2005)

Shabana, A.A.: Dynamics of Multibody Systems, 4th edn. Springer, Berlin (2013)

Sommerfeld, A.: Vorlesungen über theoretische Physik, Band 1: Mechanik, Akademische Verlagsgesellschaft, Leipzig (1948)

Terze, Z., Müller, A., Zlatar, D.: Lie-group integration method for constrained multibody systems in state space. Multibody Syst. Dyn. 34(3), 275-305 (2014)

Terze, Z., Müller, A., Zlatar, D.: An angular momentum and energy conserving Lie-group integration scheme for rigid body rotational dynamics originating from Störmer-Verlet algorithm. J. Comput. Nonlinear Dyn. 10(5), 11 pages (2015)

Tzénoff, I.: Sur les équations du mouvement des systèmes matériels non holonomes. J. de Math. pures et applique (1920)

Tzénoff, I.: Sur les équations du mouvement des systèmes matériels non holonomes. Math. Ann. 91(1/2), 161-168 (1924). https://doi.org/10.1007/BF01498387

Volterra, V.: Sopra una classe di equazioni dinamiche. Atti della Reale Accademia delle Scieze, Torino 33, 451-475 (1898)

Voronets, P.: Equations of motion for nonholonomic systems. Matematicheskii Sbornik, 22(4) (1901)

Whittaker, E.T.: A Treatise on the Analytical Dynamics of Particles and Rigid Bodies, Cambridge, 1904 (available as 4th ed., Cambridge, 1988)

Woronetz, P.: Über Die Bewegung eines starren K örpers, der ohne Gleitung auf einer beliebigen Fläche rollt. Math. Ann. 70(3), 410-453 (1910)

Zenkov, D.V., Leok, M., Bloch, A. M.: Hamel's formalism and variational integrators on a sphere. In: 51st IEEE Conf. Decision and Control (CDC), Maui, HI, pp. 7504-7510 (2012)

Publisher's Note Springer Nature remains neutral with regard to jurisdictional claims in published maps and institutional affiliations. 